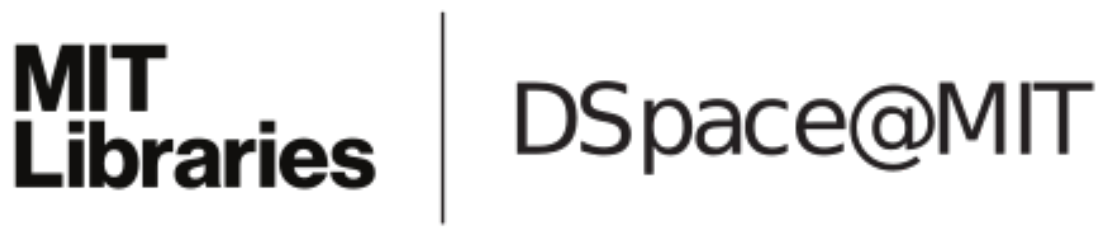

\author{
MIT Open Access Articles
}

Correlations between Black Holes and Host Galaxies in the Illustris and IllustrisTNG Simulations

The MIT Faculty has made this article openly available. Please share how this access benefits you. Your story matters.

As Published: $10.3847 / 1538-4357 / A B 8 F 8 D$

Publisher: American Astronomical Society

Persistent URL: https://hdl.handle.net/1721.1/135209

Version: Final published version: final published article, as it appeared in a journal, conference proceedings, or other formally published context

Terms of Use: Article is made available in accordance with the publisher's policy and may be subject to US copyright law. Please refer to the publisher's site for terms of use. 


\title{
Correlations between Black Holes and Host Galaxies in the Illustris and IllustrisTNG Simulations
}

\author{
Yuan $\mathrm{Li}^{1,2}$ (1D), Melanie Habouzit ${ }^{1}$ (D) , Shy Genel $^{1,3}$ (D), Rachel Somerville ${ }^{1}$, Bryan A. Terrazas ${ }^{4}$ (D), Eric F. Bell ${ }^{4}$ (D), \\ Annalisa Pillepich $^{5}$ (i), Dylan Nelson ${ }^{6}$, Rainer Weinberger ${ }^{7}$ (i), Vicente Rodriguez-Gomez ${ }^{8}$, Chung-Pei Ma ${ }^{2}(\mathbb{1})$, Ruediger Pakmor ${ }^{6}$, \\ Lars Hernquist $^{7}$ (D), and Mark Vogelsberger ${ }^{9}$ \\ ${ }^{1}$ Center for Computational Astrophysics, Flatiron Institute, 162 5th Avenue, New York, NY 10010, USA; yuan.astro@berkeley.edu \\ ${ }^{2}$ Department of Astronomy, University of California, Berkeley, CA 94720, USA \\ ${ }^{3}$ Columbia Astrophysics Laboratory, Columbia University, 550 West 120th Street, New York, NY 10027, USA \\ ${ }^{4}$ Department of Astronomy, University of Michigan, Ann Arbor, MI 48109, USA \\ ${ }^{5}$ Max-Planck-Institut für Astronomie, Königstuhl 17, D-69117 Heidelberg, Germany \\ ${ }^{6}$ Max Planck Institute for Astrophysics, Karl-Schwarzschild-Str. 1, D-85741 Garching bei Munchen, Germany \\ ${ }^{7}$ Institute for Theory and Computation, Harvard-Smithsonian Center for Astrophysics, 60 Garden Street, Cambridge, MA 02138, USA \\ ${ }^{8}$ Instituto de Radioastronomía y Astrofísica, Universidad Nacional Autónoma de México, Apdo. Postal 72-3, 58089 Morelia, Mexico \\ ${ }^{9}$ Department of Physics, Kavli Institute for Astrophysics and Space Research, Massachusetts Institute of Technology, Cambridge, MA 02139, USA \\ Received 2019 September 27; revised 2020 April 20; accepted 2020 April 30; published 2020 June 2
}

\begin{abstract}
We study black hole-host galaxy correlations, and the relation between the overmassiveness (the distance from the average $M_{\mathrm{BH}}-\sigma$ relation) of supermassive black holes (SMBHs) and the star formation histories of their host galaxies in the Illustris and TNG100 simulations. We find that both simulations are able to produce black hole scaling relations in general agreement with observations at $z=0$, but with noticeable discrepancies. Both simulations show an offset from the observations for the $M_{\mathrm{BH}}-\sigma$ relation, and the relation between $M_{\mathrm{BH}}$ and the Sérsic index. The relation between $M_{\mathrm{BH}}$ and stellar mass $M_{*}$ is tighter than the observations, especially for TNG100. For massive galaxies in both simulations, the hosts of overmassive SMBHs (those above the mean $M_{\mathrm{BH}^{-}} \sigma$ relation) tend to have larger Sérsic indices and lower baryon conversion efficiency, suggesting a multidimensional link between SMBHs and the properties of their hosts. In Illustris, the hosts of overmassive SMBHs have formed earlier and have lower present-day star formation rates, in qualitative agreement with the observations for massive galaxies with $\sigma>100 \mathrm{~km} \mathrm{~s}^{-1}$. For low-mass galaxies, such a correlation still holds in Illustris but does not exist in the observed data. For TNG100, the correlation between SMBH overmassiveness and star formation history is much weaker. The hosts of overmassive SMBHs generally have consistently larger star formation rates throughout history. These galaxies have higher stellar mass as well, due to the strong $M_{\mathrm{BH}}-M_{*}$ correlation. Our findings show that simulated SMBH scaling relations and correlations are sensitive to features in the modeling of SMBHs.
\end{abstract}

Unified Astronomy Thesaurus concepts: Supermassive black holes (1663); Galaxies (573); Star formation (1569); Galaxy evolution (594)

\section{Introduction}

It is widely accepted that the growth of supermassive black holes (SMBHs) is linked to the evolution of their host galaxies, and that SMBHs also influence the hosts via active galactic nucleus (AGN) feedback (see review by McNamara \& Nulsen 2007; Fabian 2012; Kormendy \& Ho 2013, and references therein). Depending on the level of accretion (Churazov et al. 2005), the forms of AGN feedback can be radiation, winds, jets, or a combination of them.

At high accretion rates, the accretion disk around the SMBH is radiatively efficient, and the feedback is in the so-called quasar mode or radiative mode. Observational evidence for quasar-mode feedback includes broad absorption line (BAL) winds seen close to the nuclei (Chartas et al. 2003; Moe et al. 2009; Tombesi et al. 2015; Jiang et al. 2018), and outflows on kiloparsec or even larger galactic scales (Dunn et al. 2010; Cano-Díaz et al. 2012; Arav et al. 2013; Carniani et al. 2015; Choi et al. 2015; Feruglio et al. 2015). The main wind-driving mechanism is still under debate, but many ideas have been explored from small-scale to cosmological-zoom simulations (Proga 2007; Hopkins \& Elvis 2010; Choi et al. 2012; Liu et al. 2013; Costa et al. 2018). In addition to winds, the radiation itself also has an impact on the surrounding gas (Park \& Ricotti 2012; Qiu et al. 2019).

At low accretion rates, the accretion disk around the SMBH is radiatively inefficient (Yuan \& Narayan 2014), and feedback from SMBHs is thought to be mostly related to the relativistic jets (Blandford et al. 2019), but radiation can still have an effect (Xie et al. 2017). These SMBHs are often observed as bright radio sources. Thus, this mode of feedback is often called radio-mode feedback. Radio-mode feedback is often observed in the center of cool-core galaxy clusters and massive galaxies, and is thought to be the key solution to the cooling flow problem (Fabian 1994). In other words, radio-mode feedback is generally considered responsible for maintaining the quiescent state of massive galaxies in today's universe (Schawinski et al. 2007). Thus, radio-mode feedback is also referred to as the maintenance mode. The effect of radio-mode feedback is well observed in nearby galaxy clusters and group centrals, in the form of X-ray "bubbles" (cavities) often filled with radioemitting plasma and "ripples" that are interpreted as shock waves or sound waves (e.g., Fabian et al. 2006; Wise et al. 2007; Baldi et al. 2009; Blanton et al. 2011). The energy associated with the cavities and waves is usually enough to offset radiative cooling in these systems (Dunn \& Fabian 2006; 
Rafferty et al. 2006). Optical observations have also detected kinematic signatures of fast winds from host galaxies of lowluminosity SMBHs (Cheung et al. 2016; Penny et al. 2018). Radio-mode feedback is often modeled as kinetic energy injection in numerical simulations (e.g., Dubois et al. 2010; Li \& Bryan 2014; Martizzi et al. 2019). Hence, it is also referred to as kinetic-mode feedback, even though quasar winds also carry kinetic energy, and some quasars (radio-loud quasars) are thought to produce jets, too (Wilson \& Colbert 1995; Kellermann et al. 2016).

The physical process of AGN feedback is rather complicated, but small-scale general-relativistic magnetohydrodynamic simulations have made remarkable progress in the past few years on both accretion disk physics and the launching of the jets/winds (Fragile et al. 2007; Tchekhovskoy et al. 2011; $\mathrm{Bu}$ et al. 2016; Jiang et al. 2019). Galactic-scale and cosmological-zoom simulations have also explored different ways of implementing AGN feedback, studied the effects of different feedback mechanisms, and improved our understanding of the complex interplay between galactic weather, star formation, and black hole activities (Omma et al. 2004; Guo \& Mathews 2011; Choi et al. 2012; Li et al. 2015; Yang \& Reynolds 2016; Qiu et al. 2019; Yuan et al. 2018).

Another line of evidence supporting the idea of coevolving galaxies and SMBHs is the observed black hole-host galaxy correlations. Among all black hole scaling relations, the most famous is the tight correlation between the mass of the SMBH $M_{\mathrm{BH}}$ and the stellar velocity dispersion $\sigma$ of the bulge of the host galaxy (Ferrarese \& Merritt 2000; Gebhardt et al. 2000; Greene et al. 2010; Kormendy \& Ho 2013; McConnell \& Ma 2013; Woo et al. 2013). However, $M_{\mathrm{BH}}-\sigma$ is not the only tight scaling relation. $M_{\mathrm{BH}}$ is also found to correlate with the luminosity and mass of the bulge (Kormendy 1993; Magorrian et al. 1998; Gültekin et al. 2009). Graham et al. (2001) find that $M_{\mathrm{BH}}$ also positively correlates with the concentration of the bulge, and the scatter is comparable to $M_{\mathrm{BH}}-\sigma$ or even smaller. $M_{\mathrm{BH}}$ also correlates with the total stellar mass of the host galaxy, but the scatter is much larger than $M_{\mathrm{BH}^{-}}-M_{\text {bulge }}$ (Reines \& Volonteri 2015; Terrazas et al. 2016; Shankar et al. 2019). In addition, $M_{\mathrm{BH}}$ has also been found to correlate with the spiral arm pitch angle in disk galaxies (Seigar et al. 2008; Berrier et al. 2013).

The interpretation of black hole scaling relations has been a subject of debate and may not require coevolution at all according to the central limit theorem (Hirschmann et al. 2010; Jahnke \& Macciò 2011). It has also been suggested that black hole-galaxy correlations can be achieved in simulations without AGN feedback or self-regulation (Anglés-Alcázar et al. 2013).

Recent observational studies by Martín-Navarro et al. (2016, 2018) and Terrazas et al. (2016) suggest that SMBHs are linked to the quiescent state of the host galaxies. That is, SMBHs above the mean scaling relations (Terrazas et al. 2016 use $M_{\mathrm{BH}}-M_{*}$ and Martín-Navarro et al. 2018 use $M_{\mathrm{BH}}-\sigma$ ) tend to reside in galaxies that are more "quenched" (with lower star formation rate). Martín-Navarro \& Mezcua (2018) also point out that such a correlation does not exist for smaller galaxies, suggesting that AGN feedback is less important in smaller systems.

Large-scale cosmological simulations usually employ simplistic subgrid models for AGN feeding and feedback due to the limits of resolution and computational expense.
Nonetheless, today's state-of-the-art cosmological simulations are able to recover many observed galaxy correlations and properties, such as the present-day stellar mass function and galaxy color bimodality (Schaye et al. 2015; Nelson et al. 2018; Davé et al. 2019). Many simulations have also been shown to successfully reproduce some of the black hole-host galaxy scaling relations in good agreement with observations (Sijacki et al. 2015; McAlpine et al. 2017; Weinberger et al. 2018). The relationship between SMBHs and their host properties carries a lot of information, but it is not clear how those relationships inform us about the physical processes that connect SMBHs and their hosts.

In this paper, we use the Illustris (Genel et al. 2014; Vogelsberger et al. 2014a, 2014b) and TNG100 (Marinacci et al. 2018; Naiman et al. 2018; Nelson et al. 2018; Pillepich et al. 2018a; Springel et al. 2018) simulations to study black hole scaling relations, and the relation between the overmassiveness of the SMBH and the star formation history of the host galaxy. The goal of our study is to better understand how simulated black hole scaling relations are related to subgrid models of black hole physics, and to gain more insights into how SMBHs are related to the star formation history of their host galaxies. We connect the results with those determined by selected observational data sets, albeit taken at face value and without correcting for observational selection biases.

The paper is structured as follows: Section 2 describes the key aspects of the Illustris and TNG100 simulations; in Section 3, we present the black hole-host galaxy scaling relations in Illustris and TNG100; in Section 4, we discuss how the the overmassiveness of the SMBHs is related to the quenching of their host galaxies, and compare what we find for Illustris and TNG100 with the findings in Martín-Navarro et al. (2018) and Martín-Navarro \& Mezcua (2018). We conclude this work in Section 5.

\section{Simulations}

The Illustris and TNG100 simulations are large-scale cosmological simulations that include prescriptions for star formation, stellar evolution and feedback, black hole formation, growth and feedback, and other physical processes relevant to the formation and evolution of galaxies. Both simulations are performed using the moving mesh code AREPO (Springel 2010; Pakmor et al. 2016). Illustris is an $N$-body/hydrodynamical simulation and TNG100 also includes magnetohydrodynamics. TNG100 is one of the flagship runs of the IllustrisTNG project, a successor to the original Illustris simulation, with an improved numerical scheme and updated subgrid models. The most notable changes are the revised implementation of galactic winds (stellar feedback) and a new black hole feedback model at low accretion rates. More detailed descriptions of the subgrid models can be found in Vogelsberger et al. (2013) for Illustris and in Pillepich et al. (2018b) and Weinberger et al. $(2017,2018)$ for the IllustrisTNG model adopted in TNG100. For the purpose of this work, we summarize the key aspects of the subgrid models related to SMBHs used in Illustris and TNG100.

Three important physical processes related to SMBHs are seeding, feeding, and feedback. The seeding of SMBHs in Illustris and TNG100 is similar, but the seed masses are quite different. In Illustris, an SMBH particle with a mass of $1.42 \times 10^{5} M_{\odot}$ is seeded in all halos above $7.1 \times 10^{10} M_{\odot}$ that do not already contain an SMBH. In TNG100, halos above 
$7.38 \times 10^{10} M_{\odot}$ are seeded with SMBHs of $1.18 \times 10^{6} M_{\odot}$, almost an order of magnitude larger than the seed mass in Illustris.

The accretion onto SMBHs (feeding) in both Illustris and TNG100 is the Eddington-limited Bondi accretion: $\dot{M}_{\mathrm{SMBH}}=$ $\min \left(\dot{M}_{\text {Bondi }}, \dot{M}_{\text {Edd }}\right)$. The details differ in two important aspects. First, Illustris uses a "boosted Bondi rate" with an artificial boosting factor $\alpha=100$, while TNG100 does not. From a practical point of view, boosted Bondi accretion is commonly used in cosmological simulations to grow SMBHs efficiently (e.g., Springel et al. 2005; Khalatyan et al. 2008). The physical explanation for a boosted Bondi rate is that cosmological simulations do not resolve Bondi radii, and the Bondi accretion rate computed at the actual Bondi radius is likely higher than that computed based on the gas properties at larger radii. Multiphase gas is also poorly resolved in cosmological simulations, and a boosting factor can account for the accretion of cold gas (Booth \& Schaye 2009). TNG100 does not need the boosting factor due to the large $\mathrm{SMBH}$ seeds. The second difference in the black hole growth scheme is that in Illustris, the properties of the gas that determine the Bondi formula are estimated by using only the parent gas cell where the black hole is positioned, while TNG100 uses a kernel-weighted average over neighboring cells.

Feedback from SMBHs is, in both cases, divided into two modes based on the accretion rate normalized by the Eddington rate (often referred to as the Eddington ratio): quasar mode and radio mode (Churazov et al. 2005). When the accretion rate is high, the radiative efficiency is high and the SMBH is in the quasar mode (sometimes also referred to as the "radiative mode" or "thermal mode" in the literature). In both Illustris and TNG100, this quasar-mode feedback is modeled as a continuous injection of thermal energy into a number of surrounding cells. ${ }^{10}$ The energy injection rate is $\dot{E}_{\text {therm }}=0.01 \dot{M} c^{2}$ in Illustris and $\dot{E}_{\text {therm }}=0.02 \dot{M} c^{2}$ in TNG100. The feedback from SMBH at low accretion rates is often referred to as the radio mode (sometimes also referred to as "maintenance," "jet," or "wind mode") and is modeled very differently in Illustris and TNG100. Because there is no assumption of radio emission in either Illustris or TNG100, we refer to this mode as "lowstate mode" throughout the rest of the paper. Low-state-mode feedback in Illustris is modeled by injecting thermal energy into hot bubbles at some distance from the SMBH. In TNG100, low-state-mode feedback is modeled by injecting pulsed kinetic energy into a number of cells near the SMBHs in a randomly oriented direction. The kinetic luminosity is $\dot{E}_{\text {kinetic }}=\dot{M} c^{2} \times$ $\min \left(\rho / 0.05 \rho_{\mathrm{SF}}, 0.2\right)$, where $\rho_{\mathrm{SF}}$ is the star formation threshold density. Another unique feature in TNG100 is that the dividing line between quasar-mode and low-state-mode feedback is no longer a fixed fraction of the Eddington ratio, as is the case in Illustris and most of the other cosmological simulations. Instead, the dividing line is also a function of the black hole mass:

$$
\chi=\min \left[0.002\left(\frac{M_{\mathrm{BH}}}{10^{8} M_{\odot}}\right)^{2}, 0.1\right] .
$$

As a result of this feature, SMBHs in TNG100 are mostly in the quasar (thermal) mode when their mass is much lower than

\footnotetext{
${ }^{10}$ For Illustris-1 and TNG100-1, the number of cells is 256 . We use the highest resolution simulations with $100 \mathrm{Mpc}$ boxes from both suites (Illustris-1 and TNG100-1) in this study.
}

$10^{8} M_{\odot}$, and transition to mostly low-state (kinetic) mode at $\gtrsim 10^{8} M_{\odot}$.

In both Illustris and TNG100, the implementation of SMBH feedback is inspired by the $M_{\mathrm{BH}}-\sigma$ relation or previous studies on the relation (Springel et al. 2005). However, neither Illustris nor TNG100 is specifically tuned to reproduce exactly all known black hole-host galaxy correlations. The relation between black hole mass and galaxy stellar mass (Magorrian et al. 1998) has been taken into consideration in both cases during the model development, but no exact value of the model parameters had been chosen so that the model outcome could fit precisely any specific relation (e.g., the Kormendy \& Ho 2013 relation). Thus, all the correlations studied in this work can be considered as an emerging outcome of the simulations.

IllustrisTNG (www.tng-project.org) is a suite of simulations that have boxes of different sizes and resolutions. For this work, we exclusively work with the highest resolution realization of a $\sim 100^{3}$ comoving $\mathrm{Mpc}^{3}$ box. We make the same choice for Illustris: in fact, TNG100 and Illustris have the same resolution and initial conditions, and are both evolved from $z=127$ to $z=0$. For Illustris, all the analyses are limited to galaxies with a stellar mass larger than $\sim 10^{10} M_{\odot}$ (the details of the catalog can be found in Xu et al. 2017). For TNG100, the selection criterion is stellar masses larger than $\sim 5 \times 10^{9} M_{\odot}$ within the central $30 \mathrm{kpc}$ (using the catalog from $\mathrm{Xu}$ et al. 2019). The total number of galaxies in the Illustris and TNG100 catalogs is 6808 and 9686, respectively. The catalogs in $\mathrm{Xu}$ et al. $(2017,2019)$ were originally made for different observational comparison purposes and thus have different mass cuts. We have experimented with the same mass cut at $10^{10} M_{\odot}$ for TNG100. This does not change our main findings, but cuts out a significant fraction of galaxies with low black hole masses. Because TNG100 galaxies tend to have slightly lower stellar masses on average, a slightly lower mass cut for TNG100 actually produces a more equivalent sample. Thus, we decide to keep the original sample with different mass cuts for the two simulations.

The measurements of the physical quantities of simulated galaxies are summarized in Table 1. More details are discussed in the rest of the paper.

\section{SMBH-Host Galaxy Correlations}

In this section, we discuss the black hole-host galaxy correlations in both Illustris and TNG100.

Figure 1 shows the $M_{\mathrm{BH}}-\sigma$ relation (left panels), the $M_{\mathrm{BH}}-M_{*}$ relation (middle panels), and the relation between $M_{\mathrm{BH}}$ and the Sérsic index $n$ of the host galaxy (right panels) for both Illustris (top) and TNG100 (bottom).

\subsection{The $\mathrm{M}_{B H}-\sigma$ Relation}

For comparison, we have plotted the best-fitting $M_{\mathrm{BH}^{-}}-\sigma$ relations from the observations by Kormendy \& Ho (2013) and McConnell \& Ma (2013) for all galaxies, and those by MartínNavarro et al. (2018) for the massive galaxies ${ }^{11}$ with $\sigma>100 \mathrm{~km} \mathrm{~s}^{-1}$ in the left panels of Figure 1. The sample in Martín-Navarro et al. (2018) is selected from van den Bosch (2016). Overall, both simulations are able to produce the general trend in agreement with the observations. The massive

\footnotetext{
${ }^{11}$ In this paper, we generally refer to galaxies with $\sigma>100 \mathrm{~km} \mathrm{~s}^{-1}$ as massive galaxies and galaxies with $\sigma<100 \mathrm{~km} \mathrm{~s}^{-1}$ as low-mass galaxies.
} 
Table 1

Summary of Analysis Methods and Measurements of Galaxy Properties

\begin{tabular}{|c|c|c|}
\hline Properties & Observations & Simulations in this work \\
\hline$M_{\mathrm{BH}}$ & $\begin{array}{l}\text { Kormendy \& Ho (2013), McConnell \& Ma (2013), Martín-Navarro } \\
\text { et al. (2018) }\end{array}$ & Sum of the masses of all black holes in the halo \\
\hline$M_{*}$ & Reines \& Volonteri (2015), Terrazas et al. (2016) & $\begin{array}{l}\text { Sum of all star particle masses within twice the stellar half-mass- } \\
\text { radius }\end{array}$ \\
\hline$\sigma$ & $\begin{array}{l}\text { Kormendy \& Ho (2013), McConnell \& Ma (2013), Martín-Navarro } \\
\text { et al. (2018) }\end{array}$ & $\begin{array}{l}\text { Rest-frame SDSS } r \text {-band luminosity-weighted stellar line-of-sight } \\
\text { velocity dispersion measured within a projected radius of } 1.15 \\
\text { from galaxy center in } x \text { projection (Xu et al. 2017, 2019) }\end{array}$ \\
\hline Sérsic index & Graham \& Driver (2007); Savorgnan (2016); Davis et al. (2019) & $\begin{array}{l}\text { Synthetic images designed to match Pan-STARRS observations } \\
\text { (Rodriguez-Gomez et al. 2019). }\end{array}$ \\
\hline $\begin{array}{l}\text { star formation } \\
\text { history }\end{array}$ & $\begin{array}{l}\text { Measured by Martín-Navarro et al. (2018) using the STECKMAP } \\
\text { code (Ocvirk et al. 2006) with the MILES (Vazdekis et al. 2010) } \\
\text { stellar population synthesis models }\end{array}$ & $\begin{array}{l}\text { Computed based on the formation histories of all the star particles } \\
\text { that reside within the galaxy at } z=0 \text {. We use } 0.5 R_{e} \text { for massive } \\
\text { galaxies and } 4.5 \mathrm{kpc} \text { for low-mass galaxies. }\end{array}$ \\
\hline
\end{tabular}

galaxies in Illustris lie above the observed $M_{\mathrm{BH}}-\sigma$ relations, but the slope is roughly consistent. At the low-mass end, $M_{\mathrm{BH}^{-}}-\sigma$ in Illustris bends down and becomes closer to the observations, although Martín-Navarro \& Mezcua (2018) suggest that the observed relation bends up (the slope is shallower) for the lowmass galaxies. The massive galaxies in TNG100 are also above the observations (except for some of the very massive ones). The linear best fit (the red line) has a slope that is smaller than the observed slope, but it is also obvious that the $M_{\mathrm{BH}}-\sigma$ relation in TNG100 cannot be well described with a linear fit. The relation appears to have a break at $\log _{10} \sigma\left(\mathrm{km} \mathrm{s}^{-1}\right) \sim 2.1$, and from there to $\log _{10} \sigma\left(\mathrm{km} \mathrm{s}^{-1}\right) \sim 1.9$, the relation flattens. This also corresponds to a black hole mass of $\gtrsim 10^{8} M_{\odot}$, which is the critical mass that helps determine feedback modes, as described in Section 2. The relation steepens again at $\log _{10} \sigma\left(\mathrm{km} \mathrm{s}^{-1}\right)<1.9$, but quickly flattens at the low $-\sigma$ end to $\log _{10} M_{\mathrm{BH}}\left(M_{\odot}\right) \sim 7.5$.

It is unlikely that the discrepancy between simulations and observations can be attributed to the different ways galaxy properties are measured. For both Illustris and TNG100, we use $\sigma_{x}$, which is the rest-frame SDSS $r$-band luminosity-weighted stellar line-of-sight velocity dispersion measured within a projected radius of 1 !" 5 from galaxy center in $x$ projection $(\mathrm{Xu}$ et al. 2017, 2019). As there is no preferred direction in Illustris or TNG100, projections along the $y$ - and $z$-axes are the same and we have verified this. We have also tried to use $\sigma$ measured within $0.5 R_{e}$ and $2.0 R_{e}$ and found very little change. If we use the stellar-mass-weighted $\sigma$ instead of the light-weighted $\sigma$, the $M_{\mathrm{BH}}-\sigma$ relation steepens very slightly, but the general results are consistent. The Illustris $M_{\mathrm{BH}}-\sigma$ also appears consistent with the relation presented in Sijacki et al. (2015), which independently computed $\sigma$ directly from the simulation data.

The main discrepancy between simulations and observations for both Illustris and TNG100 is that on the $M_{\mathrm{BH}}-\sigma$ diagram, the simulated galaxies are above the observed $M_{\mathrm{BH}}-\sigma$ relation (Figure 1). This is possibly due to SMBHs being overly massive in simulations and/or $\sigma$ being too low. The black hole mass function in Illustris is in good agreement with the observations (Sijacki et al. 2015) and the TNG100 quasar luminosity function at low redshift also agrees well with the observations (Habouzit et al. 2019; Weinberger et al. 2018). This suggests that at least in the local universe, at the high-mass end, SMBHs in these simulations are generally not too massive. Thus, overly massive
SMBHs cannot be the main reason for the order of magnitude offset in the $M_{\mathrm{BH}}-\sigma$ relation.

At the high-mass end, the discrepancy between the $M_{\mathrm{BH}^{-}}-\sigma$ relations for Illustris and massive galaxies in TNG100 likely has to do with $\sigma$ being too small in simulations. Figure 2 shows the relation between $\sigma$ and total stellar mass of a galaxy (Faber Jackson Relation) in Illustris (top) and TNG100 (bottom). We also separate early-type and late-type galaxies based on their Sérsic indices. Strictly, the Faber Jackson Relation only applies to early-type galaxies. Because there are very few galaxies with Sérsic index $>4$ in Illustris, and because simulations may underpredict Sérsic indices, we show both early- and late-type galaxies in Figure 2. In both simulations, for a given $M_{*}, \sigma$ tends to be lower than the observed relation, and the disagreement is worse for lower mass galaxies. The best agreement is found between observations and massive galaxies in TNG100. The Illustris Faber Jackson Relation is discussed in more detail in $\mathrm{Xu}$ et al. (2017), which also shows that the sizes of the Illustris galaxies tend to be too large. For massive galaxies in TNG100, Genel et al. (2018) find that the effective radius is overestimated by $0.1-0.15 \mathrm{dex}$ at $M_{*}>10^{10.5} M_{\odot}$ (or up to 1.5 times; see also Wang et al. 2020; Rodriguez-Gomez et al. 2019). For a given mass, $\sigma$ and $R_{e}$ are roughly related to each other as $\sigma^{2} \sim 1 / R_{e}$. Thus, a $0.1-0.2$ dex difference in $R_{e}$ corresponds to a $0.05-0.1$ dex difference in $\log _{10} \sigma\left(\mathrm{km} \mathrm{s}^{-1}\right)$ and can explain the discrepancy seen in Figure 1 for massive galaxies. The better agreement in Faber Jackson Relation for TNG100 galaxies with $\log _{10} \sigma\left(\mathrm{km} \mathrm{s}^{-1}\right)>2.2$ is also consistent with our finding that the massive galaxies in TNG100 have a better agreement in the $M_{\mathrm{BH}}-\sigma$ relation. In Illustris, the sizes of galaxies are even more overestimated, about twice as large as the observed galaxies on average for all the galaxies we study here $\left(M_{*}>10^{10} M_{\odot}\right.$; Snyder et al. 2015; Bottrell et al. 2017). This is consistent with Figure 2, which shows that for a given $M_{*}$, the offset in $\sigma$ is larger for Illustris than for TNG100. A factor of 2 difference in $R_{e}$ corresponds to a 0.15 dex shift in $\log _{10} \sigma\left(\mathrm{km} \mathrm{s}^{-1}\right)$, which is again consistent with the offset we find in the Illustris $M_{\mathrm{BH}}-\sigma$ relation in Figure 1.

For low-mass galaxies $\left(\log _{10} \sigma\left(\mathrm{km} \mathrm{s}^{-1}\right)<2.0\right)$, the galaxy sizes in TNG100 agree well with the observations (Genel et al. 2018), and the offset in $\sigma$ shown in Figure 2 is not enough to explain the discrepancy we see in the TNG $M_{\mathrm{BH}^{-}}-\sigma$ relation for low-mass galaxies. Therefore, in low-mass galaxies, SMBHs in TNG100 are likely too massive. Two factors may contribute to 

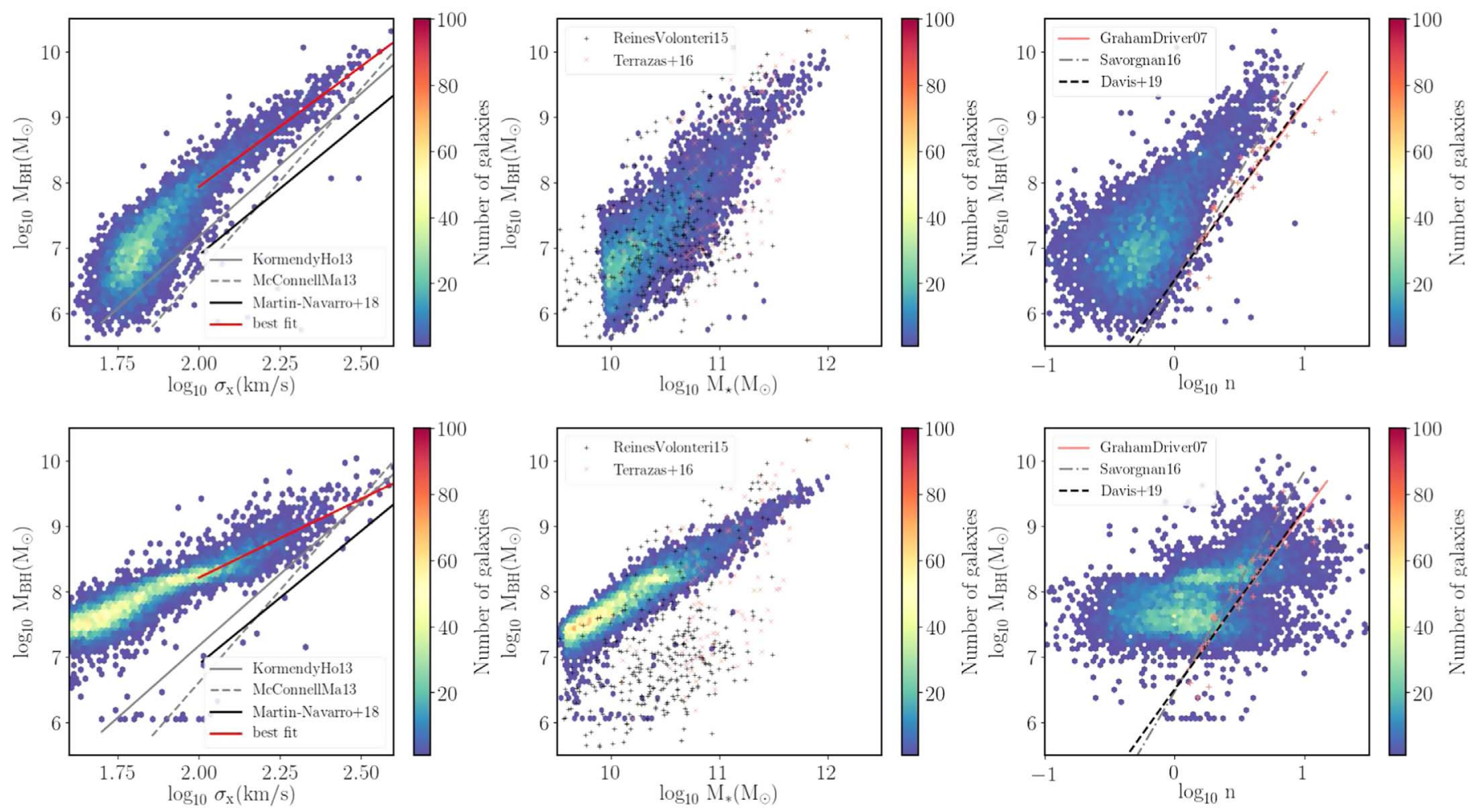

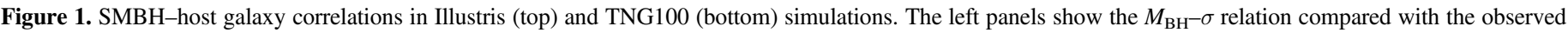

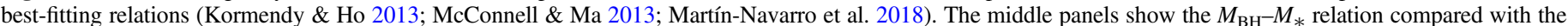

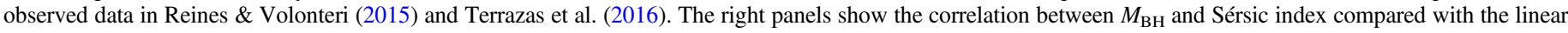

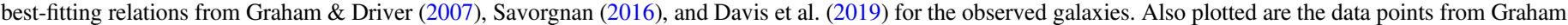

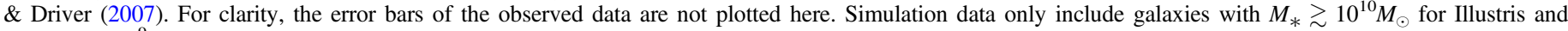
$M_{*} \gtrsim 5 \times 10^{9} M_{\odot}$ for TNG100.

this discrepancy. First, SMBH seeds in TNG100 may be too massive, $\sim 10^{6} M_{\odot}$. Because the Bondi accretion rate scales as $M_{\mathrm{BH}}^{2}$, more massive seeds also lead to more accretion onto the SMBH. In addition, low-mass SMBHs in TNG100 tend to be in quasar mode, and the pure thermal quasar-mode feedback in TNG100 is not very effective at removing gas from the central region of the host galaxy. Thus, the growth of SMBHs in quasar mode may be too fast. The overly efficient early growth of SMBHs is also used to explain the overly abundant luminous SMBHs at high redshift in TNG100 (Weinberger et al. 2018).

\subsection{The $\mathrm{M}_{B H}-\mathrm{M}_{*}$ Relation}

The middle panels of Figure 1 show the $M_{\mathrm{BH}}-M_{*}$ relation in Illustris (top) and TNG100 (bottom). The cut at the low-mass end has to do with a cut in the catalog (see Section 2 for details). For comparison, we have also overplotted the observational data compiled in Terrazas et al. (2016) with dynamical estimates of black hole masses and Reines \& Volonteri (2015) which includes a sample of broad-line AGNs at low redshift, as well as galaxies with dynamical black hole masses. The Illustris $M_{\mathrm{BH}}-M_{*}$ relation compared with different observations has been shown in Sijacki et al. (2015) and Terrazas et al. (2016); the TNG300 $M_{\mathrm{BH}}-M_{*}$ relation is shown in Weinberger et al. (2018) and is discussed in detail in a separate paper (Terrazas et al. 2020). Here, we only focus on the key aspects that are relevant to the discussions in this work.

Both Illustris and TNG100 show correlations between the total stellar mass of a galaxy $M_{*}$ and $M_{\mathrm{BH}}$ that are very broadly in agreement with observations. At the high-mass end, simulations slightly underpredict $M_{\mathrm{BH}}$. This has also been noted in Mutlu-Pakdil et al. (2018) for Illustris central galaxies. However, the scatter is smaller than the observations. The means are offset between observations and models, too. For instance, at $\log _{10} M_{*}\left(M_{\odot}\right)=10.2$, the mean $\log _{10} M_{\mathrm{BH}}\left(M_{\odot}\right)$ in the Reines \& Volonteri (2015) sample is 6.7, with a $1 \sigma$ scatter of 0.6. The mean $\log _{10} M_{\mathrm{BH}}\left(M_{\odot}\right)$ in Illustris and TNG100 at $\log _{10} M_{*}\left(M_{\odot}\right)=10.2$ is 6.9 and 7.9 , respectively, and the scatters are $\sim 0.4$ for Illustris and 0.2 for TNG100. Models hug the high black hole mass envelope of galaxies, and do not produce the galaxies with lower black hole mass at a given stellar mass. This is especially pronounced for TNG100, which produces an $M_{\mathrm{BH}}-M_{*}$ correlation that is even tighter than the $M_{\mathrm{BH}}-\sigma$ relation.

Many factors may contribute to the difference in the amount of scatter between simulations and observations. First, due to resolution limit, simulations do not fully capture the stochasticity of black hole accretion and feedback. Second, the SMBH seeding scheme is such that the initial SMBH seed mass is tightly linked to the halo mass, which is correlated with the stellar mass. The central limit theorem (Hirschmann et al. 2010; Jahnke \& Macciò 2011) suggests that these correlations should get tighter through subsequent mergers. Third, the value of the radiative efficiency is set partly by the spin of the black hole and the geometry of the accretion flow (Bustamante \& Springel 2019), a dependence that is not included in the simulations. The same applies to the coupling efficiency, which is currently set to a fixed constant value in both simulations. It 

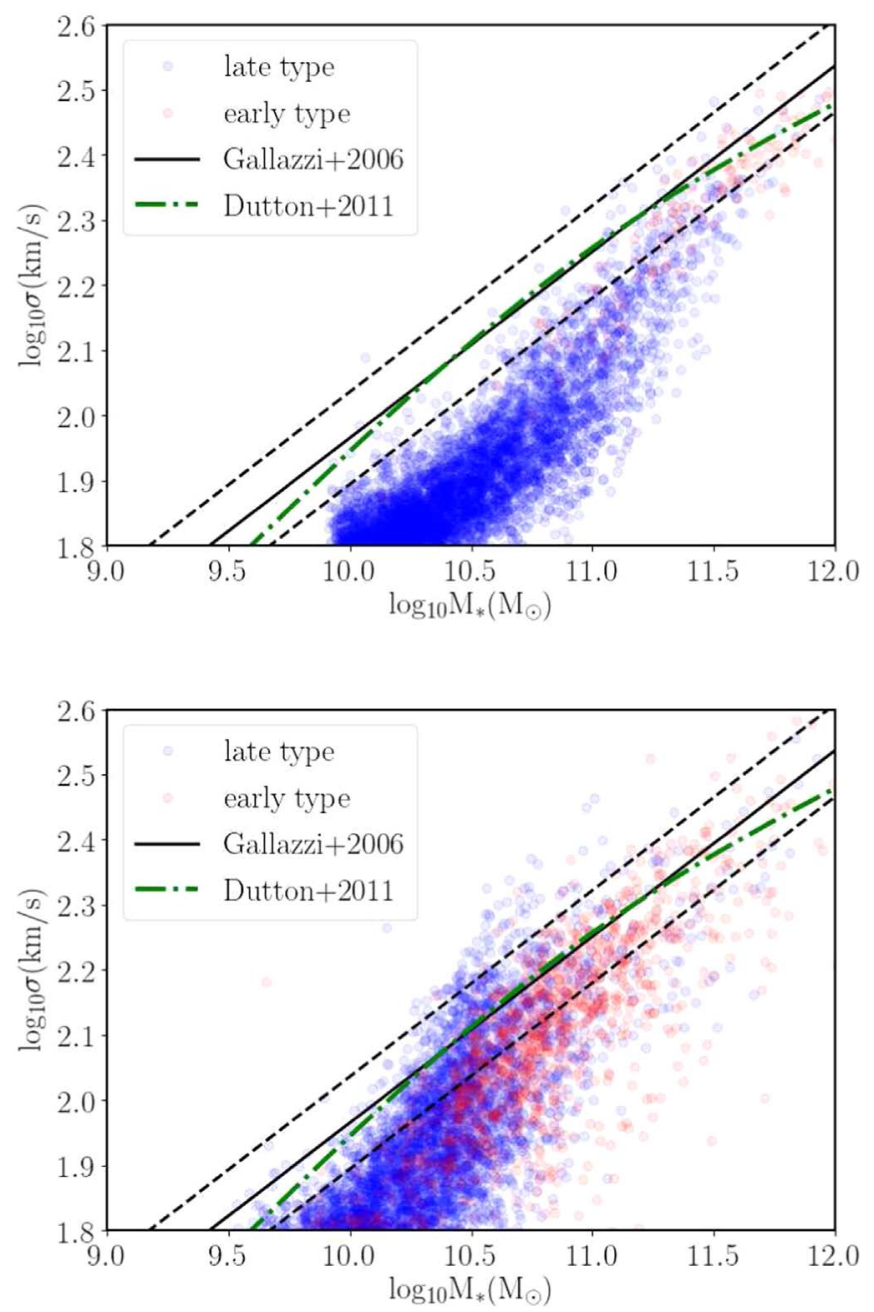

Figure 2. The relation between stellar velocity dispersion $\sigma$ and stellar mass $M_{*}$ for galaxies in Illustris (top) and TNG100 (bottom). Blue dots show latetype galaxies (defined as those with Sérsic indices smaller than 4), and red dots show early-type galaxies (Sérsic indices $\geqslant 4$ ). The solid black line is the linear fit to the Faber Jackson Relation in Gallazzi et al. (2006) for early-type galaxies with $R_{90} / R_{50}>2.8$ in the Sloan Digital Sky Survey (SDSS) Data Release 2 (Abazajian et al. 2004) and dashed lines show the scatter. The dotted-dashed green line shows the best fit for the early-type galaxies in SDSS Data Release 7 (Abazajian et al. 2009) using a double power law (Dutton et al. 2011). For a given $M_{*}, \sigma$ tends to be low in simulations, and the discrepancy is larger in Illustris. Massive galaxies in TNG100 agree reasonably well with the observations, although with a tendency toward low $\sigma$ at a given $M_{*}$.

is plausible to believe that, if a wider range of distributions in these factors had been allowed in the models, the scatter in black hole mass at a given stellar mass could have been larger. Additionally, in TNG100, the accretion rate onto SMBHs is computed using a kernel-weighted average over 256 neighboring cells. As a result, the SMBH accretion is correlated with the gas properties of a volume that makes up a non-negligible fraction of the star-forming region of the host galaxy. This may be one reason why TNG100 produces an even tighter $M_{\mathrm{BH}}-M_{*}$ correlation than Illustris.

\subsection{The $M_{\mathrm{BH}}-$ Sérsic Index Relation}

The right panels of Figure 1 show the relation between $M_{\mathrm{BH}}$ and the Sérsic index $n$ of the host galaxy which measures the concentration of light from the stars in the galaxy.
The Sérsic indices are measured using synthetic images of simulated galaxies created with the SKIRT (Baes et al. 2011) radiative transfer code, which include the effects of dust attenuation and scattering, and are designed to match PanSTARRS observations (Rodriguez-Gomez et al. 2019). For Illustris galaxies, we have compared our Sérsic indices with those obtained by fitting a 2D Sérsic profile to the radial distribution of the elliptical isophotes of mock SDSS images of simulated galaxies (Xu et al. 2017). For galaxies with low Sérsic indices, the dust-corrected Sérsic indices tend to be slightly lower than the uncorrected measurements, but the overall agreement between the two measurements is very good.

Also plotted in the right panels of Figure 1 is the best linear fit for the 27 observed galaxies in Graham \& Driver (2007). ${ }^{12}$ The data in Graham \& Driver (2007) are from Graham et al. (2001), which use SMBHs from Merritt \& Ferrarese (2001) with revised $M_{\mathrm{BH}}$ estimates from Kormendy \& Gebhardt (2001), and Sérsic indices based on high-quality $R$-band images. The actual data are plotted as pink symbols. We have also included the best linear fit lines from Savorgnan (2016) and Davis et al. (2019). Savorgnan (2016) uses 66 galaxies with dynamical measurement of $M_{\mathrm{BH}}$ and for which they are able to successfully model the light distribution and measure the spheroid structural parameters using $3.6 \mu \mathrm{m}$ Spitzer satellite images. Davis et al. (2019) measures $M_{\mathrm{BH}}-n$ relation for a sample of 40 spiral galaxies using the spheroid major axis Sérsic indices.

The $M_{\mathrm{BH}}-n$ relations in both Illustris and TNG100 show a trend in general agreement with the observations. The simulated data are above (or shifted to the left of) the observed relation. This shift is larger in Illustris than in TNG100, especially at the high-mass end. Similar to the offset in $M_{\mathrm{BH}^{-}}-\sigma$ relation, we do not think that this discrepancy is solely due to SMBHs being overly massive in simulations.

As Rodriguez-Gomez et al. (2019) show by mocking simulated galaxies as if imaged within Pan-STARRS observations, the Sérsic indices of Illustris galaxies are lower by a factor of 2-3 in comparison to Pan-STARRS. TNG100 galaxies have Sérsic indices that are in much better agreement with the Pan-STARRS observations than Illustris, and are only slightly lower at $M_{*} \sim$ a few times $10^{10} M_{\odot}$. However, because of the small range of Sérsic indices compared with the range of $M_{\mathrm{BH}}$, which spans orders of magnitude, a small mismatch in the Sérsic index can result in a large apparent discrepancy in the $M_{\mathrm{BH}}-n$ relation. According to Rodriguez-Gomez et al. (2019), at $M_{*}$ of a few times $10^{10} M_{\odot}$, the average Sérsic index of the Pan-STARRS galaxies is $\sim 3$, and the average Illustris and TNG100 Sérsic indices for the same stellar mass is about $\sim 0.7$ and 1.5-2, respectively. These correspond to a shift of $\sim 0.6$ dex and $\sim 0.2-0.3$ dex in $\log _{10} n$ for Illustris and TNG100, respectively, and are consistent with what we see in the right panel of Figure 1. Therefore, it is plausible that, if simulated galaxies had higher Sérsic indices $n$ (and thus in better agreement with Pan-STARRS data), they would better agree with the observed $M_{\mathrm{BH}^{-}}-n$ relations in Figure 1.

It has previously been suggested that if simulated galaxies underpredict $\sigma$ and Sérsic $n$, this could be due to insufficient numerical resolution (e.g., see Hopkins et al. 2018 for discussions on how resolution affects the central density profiles of simulated

\footnotetext{
12 Although a linear relation provides a good fit to the data, Graham \& Driver (2007) find that the best-fitting quadratic relation has smaller scatter. We use the linear fit here for simplicity.
} 
galaxies using the FIRE-2 physical model). However, resolution cannot be the only issue here. Illustris and TNG100 galaxies are modeled at the same mass and spatial resolution, yet they exhibit different size-mass and Sérsic index-mass relations (see Pillepich et al. 2018b; Rodriguez-Gomez et al. 2019).

As is pointed out in Graham et al. (2001) and Graham \& Driver (2007), $\log _{10} M_{\mathrm{BH}}$ and $\log _{10} n$ may not be linearly correlated. While the $M_{\mathrm{BH}}-n$ relation in Illustris could be well described with a linear relation with some scatter, this is certainly not the case for TNG100. In TNG100, there is a concentration of galaxies with $\log _{10} n \sim 0-0.3$ and $M_{\mathrm{BH}} \sim$ a few times $10^{7} M_{\odot}$, and a horizontal strip at $M_{\mathrm{BH}} \gtrsim 10^{8} M_{\odot}$. These features are consistent with what is seen in the other black hole scaling relations and are likely related to the large seed mass, the ineffective quasar-mode feedback, and the critical $M_{\mathrm{BH}}$ in the feedback-mode transition discussed previously.

\subsection{The Importance of Features in the Black Hole Scaling Relations}

As Figure 1 shows, all the TNG100 black hole-host galaxy correlations show a horizontal feature at the critical $M_{\mathrm{BH}}$ (it is less prominent in the $M_{\mathrm{BH}}-M_{*}$ relation but still noticeable). The reason may be that $\mathrm{SMBH}$ feedback is not efficient enough below $10^{8} M_{\odot}$, allowing fast growth for small SMBHs, but becomes slightly too efficient at a few times $10^{8} M_{\odot}$ (see also Habouzit et al. 2019) and starts to suppress the growth of the SMBHs themselves. As the galaxies continue to evolve, the growth of the SMBH lags behind the growth of other quantities such as $\sigma$ and $M_{*}$. This results in a pile-up of galaxies with $M_{\mathrm{BH}} \gtrsim 10^{8} M_{\odot}$. This is not necessarily wrong. Graham \& Scott (2013) suggest that there is a break in black hole scaling relations because the growth of $M_{\mathrm{BH}}$ is dominated by different processes at different masses. As discussed previously, a break in the $M_{\mathrm{BH}}-\sigma$ relation is also suggested by Martín-Navarro \& Mezcua (2018). Recent theoretical studies by Bustamante \& Springel (2019) also suggest that a transition near $M_{\mathrm{BH}} \gtrsim 10^{8} M_{\odot}$ should naturally emerge as a result of black hole spin evolution. Bustamante \& Springel (2019) also identify the same "bottleneck" effect at this specific black hole mass scale which results in an overabundance of SMBHs at around $10^{8} M_{\odot}$ at $z=0$.

Our analysis suggests that if the transition between quasarmode and low-state-mode feedback is indeed dependent on $M_{\mathrm{BH}}$ as is implemented in TNG100, we may be able to find features associated with the critical $M_{\mathrm{BH}}$ in the observed data. More specifically, if low-state-mode feedback is indeed more effective than quasar mode in nature, then black hole scaling relations should show a similar overdensity near the transition mass as is shown here.

We note that when comparing simulations with observations, quantities directly measured from the simulation data can be different from those inferred from observations. Thus, when possible, we always use the quantities measured from synthetic observations of the simulation data (e.g., $\sigma$ and Sérsic indices; see Table 1 for a summary). In addition, different observations have different selection functions, which can bias the results. For example, the best-fit $M_{\mathrm{BH}}-\sigma$ relations in Kormendy \& Ho (2013), McConnell \& Ma (2013), and Martín-Navarro et al. (2018) have different slopes and/or normalizations from each other (Figure 1). Moreover, the observed sample may not have a well-defined selection function. For example, the data in Terrazas et al. (2016) and Graham \& Driver (2007) are not from single surveys and thus the biases are hard to measure. For those reasons, comparisons between simulations and observations here are mostly done at a qualitative level.

On the other hand, the simulation data are processed in the same way for Illustris and TNG100. As we have shown, black hole-host galaxy scaling relations can be quite different between the two simulations, and much of the difference can be attributed to how black holes physics is modeled. In Section 4, we further explore how subgrid models affect the complex interplay of black hole growth, star formation history and galaxy properties.

\section{SMBH Overmassiveness and Quiescence}

The $M_{\mathrm{BH}^{-}}-\sigma$ relation is quite tight in both observations and simulations as is shown in Figure 1. However, there is still some amount of scatter (Tremaine et al. 2002; Gültekin et al. 2009; McConnell \& Ma 2013). ${ }^{13}$ Martín-Navarro et al. (2018) measure the star formation histories of nearby massive galaxies $\left(\sigma>100 \mathrm{~km} \mathrm{~s}^{-1}\right)$ from their integrated optical spectra and find that the host galaxies of overmassive SMBHs (defined as ones above the average $M_{\mathrm{BH}^{-}} \sigma$ relation) have formed earlier and have had suppressed star formation for longer. In this section, we examine the relation between SMBH overmassiveness and quiescence of host galaxies in Illustris (Section 4.1) and TNG100 (Section 4.2).

\subsection{SMBH Overmassiveness in Illustris}

\subsubsection{SMBH Overmassiveness in Massive Galaxies in Illustris}

As Figure 1 shows, there is an offset between the simulated and observed $M_{\mathrm{BH}^{-}}-\sigma$ relations. Thus, instead of using the observed $M_{\mathrm{BH}}-\sigma$ relation, we first perform a linear fit to the simulated galaxies with $\sigma>100 \mathrm{~km} \mathrm{~s}^{-1}$ and use that as the average $M_{\mathrm{BH}}-\sigma$ relation to define overmassive and undermassive black holes in the two simulations separately. The best-fitting $M_{\mathrm{BH}^{-}}-\sigma$ relation for galaxies with $\sigma>100 \mathrm{~km} \mathrm{~s}^{-1}$ in Illustris is $\log _{10} M_{\mathrm{BH}}\left(M_{\odot}\right)=3.7 \log _{10} \sigma\left(\mathrm{km} \mathrm{s}^{-1}\right)+0.56$.

As discussed in Section 3, both Illustris and TNG100 simulations likely underpredict $\sigma$ (by order of $\sim 0.1$ dex). Martín-Navarro et al. (2018) and Martín-Navarro \& Mezcua (2018) use $\sigma=100 \mathrm{~km} \mathrm{~s}^{-1}$ as the dividing line between massive and low-mass galaxies in the observed sample. When we use the same $\sigma=100 \mathrm{~km} \mathrm{~s}^{-1}$ as the dividing line, we are effectively using a slightly higher $\sigma$, corresponding to a higher $M_{\mathrm{BH}}$. We have tested using $\log _{10} \sigma\left(\mathrm{km} \mathrm{s}^{-1}\right)=1.9$ as the cut and found that the results remain qualitatively the same for for both massive and low-mass galaxies for both Illustris and TNG100. Thus, for simplicity and consistency, we use $\sigma=100 \mathrm{~km} \mathrm{~s}^{-1}$ but caution that this selection criterion is slightly different from Martín-Navarro et al. (2018) due to the difference in $\sigma$ between simulations and observations.

Figure 3 shows the comparison between the star formation histories of the massive galaxies hosting overmassive SMBHs (red) and undermassive SMBHs (blue) in Illustris. The left and middle panels show the absolute and normalized star formation rate (nSFR) as a function of lookback time and redshift. The nSFR is computed as star formation rate divided by the galaxy's current-day stellar mass, as is used in Martín-Navarro et al. (2018). This is different from specific star formation rate (sSFR) as sSFR at any given time is calculated as the star

\footnotetext{
${ }^{13}$ It has been argued that the scatter in the $M_{\mathrm{BH}}-\sigma$ relation is no larger than the measurement error alone (Ferrarese \& Merritt 2000).
} 

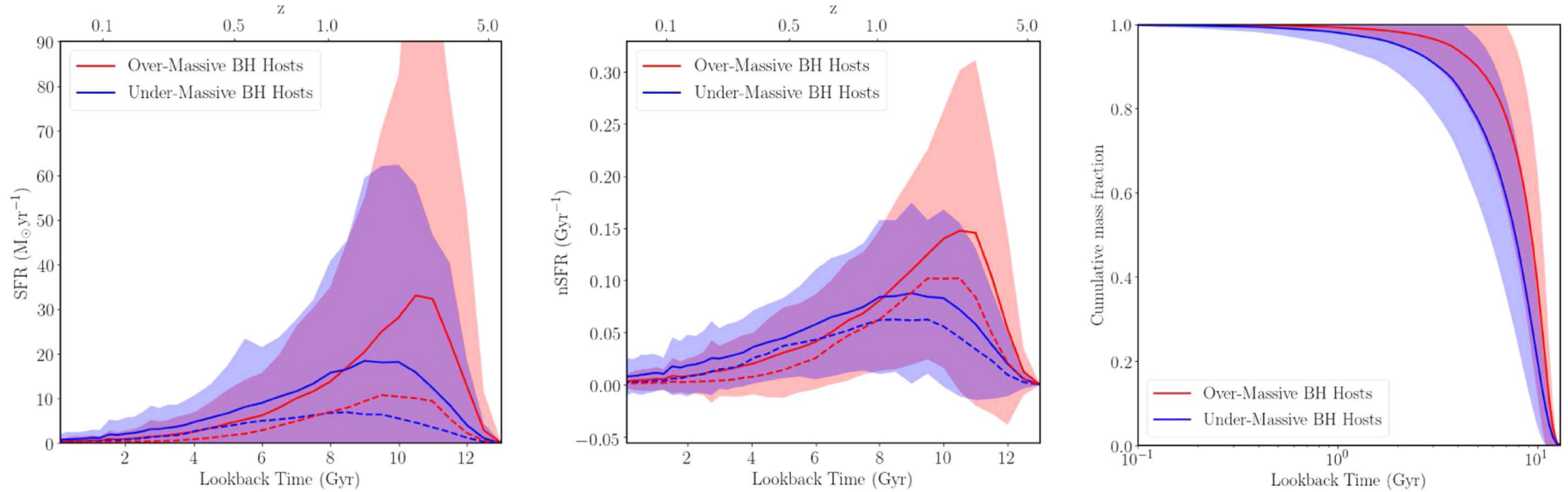

Figure 3. Star formation history of massive galaxies $\left(\sigma>100 \mathrm{~km} \mathrm{~s}^{-1}\right)$ in Illustris. The left panel shows star formation rate as a function of lookback time. Red shows the galaxies with overmassive SMBHs and blue shows galaxies with undermassive SMBHs as defined in Section 4.1. Within each lookback time bin, we fit a normal distribution to the SFR distribution of each population, and the mean is shown as solid lines. The shaded regions are the $1 \sigma$ range. The dashed lines show the median values for the two populations. The middle panel shows the star formation rate normalized by the current-day stellar mass. The right panel shows the cumulative mass fraction as a function of lookback time, color coded in the same way as the top panel. Similar to the observed massive galaxies in Martín-Navarro et al. (2018), the hosts of overmassive SMBHs have formed earlier and have had suppressed star formation for longer in Illustris.

formation rate divided by the galaxy's stellar mass at that time. The star formation rate of individual galaxies in the simulations is computed based on the formation histories of all the star particles that reside within the galaxy at $z=0$. This is different from the in situ SFR of the galaxy progenitors as our SFR also includes the star particles that formed ex situ, but were accreted later. We choose this way of computing SFR because this is the closest to what can be obtained from the spectra of the observed galaxies.

The solid lines in Figure 3 are the mean SFR within each lookback time bin for the two populations and the shaded area brackets the $1 \sigma$ deviation. The dashed lines are the median values within each bin. Whether we use the mean SFR or median SFR, the host galaxies of overmassive SMBHs have a higher SFR and nSFR at higher redshift, and lower nSFR at lower redshift. The transition happens at $z \gtrsim 1$. The right panel shows the cumulative stellar mass as a function of lookback time. Galaxies hosting overmassive SMBHs have formed their stellar mass earlier than the ones hosting undermassive SMBHs. This is in qualitative agreement with the findings in Martín-Navarro et al. (2018) for the observed massive galaxies. Even the time when the nSFRs of the two populations cross is similar. In Martín-Navarro et al. (2018), it happens about $10 \mathrm{Gyr}$ ago, and in Illustris, about $8 \mathrm{Gyr}$ ago.

Martín-Navarro et al. (2018) has a selection bias due to line contamination. Galaxies with high current-day SFR tend to be excluded from the sample, and thus the sample is heavily biased toward quiescent galaxies. In order to test the effect of this selection bias, we have experimented with only selecting Illustris galaxies with sSFR $<10^{-11} \mathrm{yr}^{-1}$ and sSFR $<10^{-12} \mathrm{yr}^{-1}$. Out of our full Illustris sample of 6808 galaxies, these two criteria select 894 and 588 galaxies, respectively. Both subsamples show the same trend as the full sample. This confirms that the result is not sensitive to the selection bias related to SFR. For completeness, we use the full sample without an sSFR cut throughout the paper. Martín-Navarro et al. (2018) also use a fixed aperture of $0.5 R_{e}$, where $R_{e}$ is the half-light radius of a galaxy. We have adopted the same $0.5 R_{e}$ aperture, but our $R_{e}$ is the half-mass radius, which is not exactly the same as the half-light radius. We have experimented with $R_{e}$ and $2 R_{\mathrm{e}}$, and found the trend to be the same. This suggests that at least for simulated massive galaxies, the results are not sensitive to the exact aperture.

One interpretation of the correlation between SMBH overmassiveness and quiescence as proposed in Martín-Navarro et al. (2018) is that quenching happens earlier and more efficiently in galaxies hosting overmassive SMBHs. We use the vertical distance to the average $M_{\mathrm{BH}^{-}}-\sigma$ as a measurement of overmassiveness of SMBHs and define the baryon conversion efficiency as the total stellar mass divided by the total halo mass. We find a statistically significant (albeit weak) negative correlation between the two quantities with a Pearson's correlation coefficient of $r=-0.12$ (with a $95 \%$ confidence interval of $[-0.18,-0.06])$ and a $p$-value of $\log _{10} p \sim-4$ (topleft panel of Figure 4).

This is also seen in the top-right panel of Figure 4, which shows the $M_{\mathrm{BH}^{-}}-\sigma$ relation weighted by the baryon conversion efficiency. The galaxies that lie above the average $M_{\mathrm{BH}}-\sigma$ tend to be less efficient in converting baryons into stars. Snyder et al. (2015) find that in Illustris, SMBHs that are overmassive with respect to the mean $M_{\mathrm{BH}}$ - halo mass relation correlate with smaller host stellar mass. The interpretation is that higher SMBH mass implies more total feedback energy and thus a greater reduction in $M_{*}$. This is in concordance with our findings here.

Sérsic indices of galaxies measure the concentration of stars in the host galaxy and are correlated with the formation time of galaxies in that older galaxies tend to have higher Sérsic indices. Given that the hosts of overmassive SMBHs have formed their stars earlier, one would expect them to have higher Sérsic indices. The bottom two panels of Figure 4 show that overmassiveness of SMBHs is indeed positively correlated with the Sérsic index of the host galaxy, with a Pearson's correlation coefficient of 0.22 and $\log _{10} p \sim-12$. This is not surprising given that $M_{\mathrm{BH}}$ is correlated with Sérsic indices as is shown in Figure 1. However, we do note that the $M_{\mathrm{BH}^{-}}-n$ relation does not necessarily mean that the overmassiveness on $M_{\mathrm{BH}^{-}} \sigma$ should be correlated with Sérsic $n$.

When we trace the history of the main progenitors of the Illustris galaxies using the SubLink merger tree (RodriguezGomez et al. 2015), we indeed find that the host galaxies of 

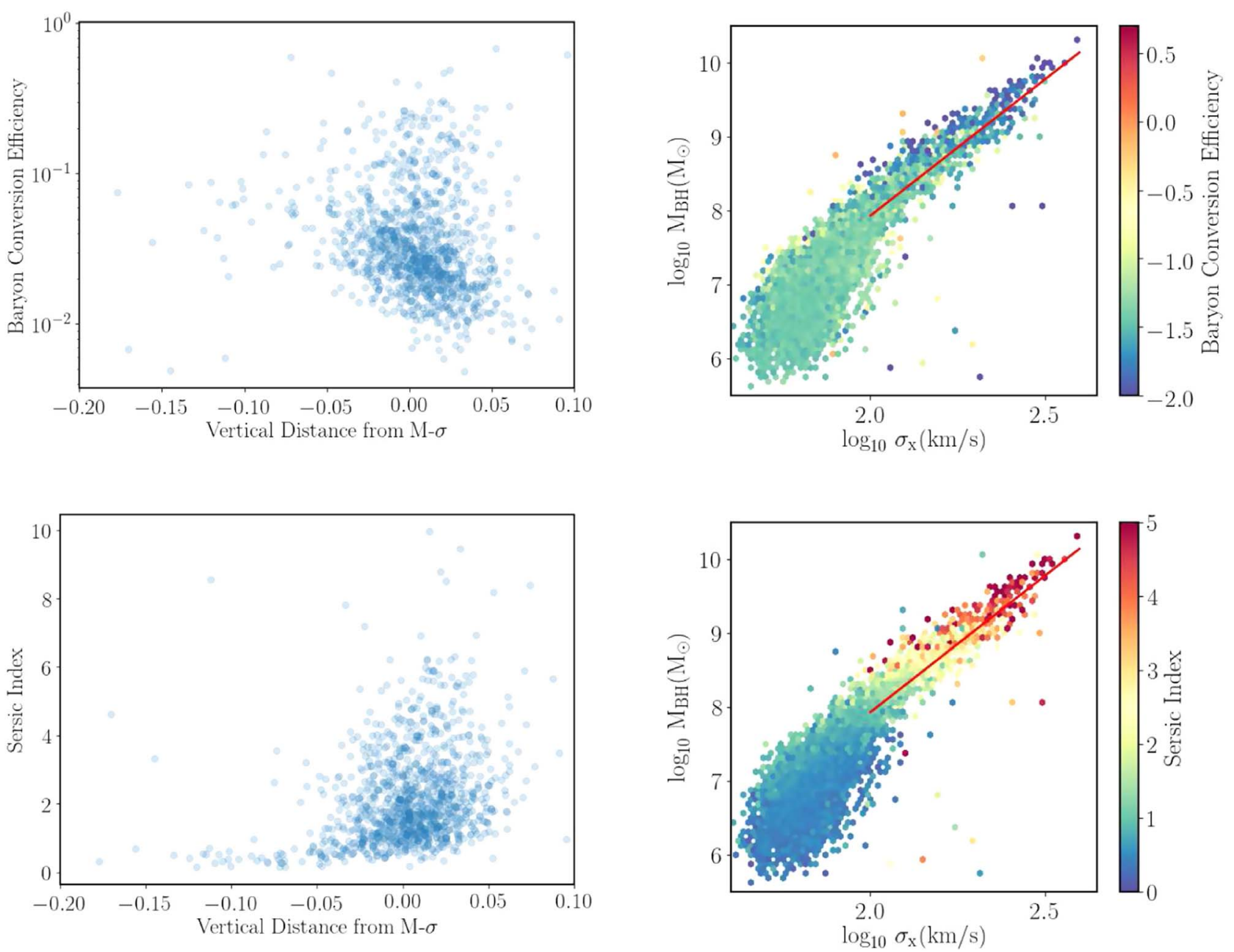

Figure 4. In Illustris, the overmassiveness of SMBHs (vertical distance from the average $M_{\mathrm{BH}^{-}} \sigma$ relation) correlates with the Sérsic indices of the host galaxies and anticorrelates with the baryon conversion efficiency. The left panels show only galaxies with $\sigma>100 \mathrm{~km} \mathrm{~s}^{-1}$ in Illustris.

overmassive SMBHs have formed earlier and on average always have a slightly higher total halo mass. Figure 5 shows that the current-day overmassive SMBHs have also formed earlier and have been more massive throughout history. There is overlap between the hosts of overmassive SMBHs and the hosts of undermassive SMBHs, but the median values (thick dashed lines in Figure 5) are well separated.

\subsubsection{SMBH Overmassiveness in Low-mass Galaxies in Illustris}

Although at $\sigma>100 \mathrm{~km} \mathrm{~s}^{-1}$ the host galaxies of overmassive SMBHs and undermassive SMBHs have different star formation histories, Martín-Navarro \& Mezcua (2018) find that for smaller galaxies with $\sigma<100 \mathrm{~km} \mathrm{~s}^{-1}$, this is not the case. The star formation history of smaller galaxies appears uncorrelated with the overmassiveness of their SMBHs. Their interpretation is that AGN feedback is not important in small galaxies with $\sigma<100 \mathrm{~km} \mathrm{~s}^{-1}$. We fit a linear $M_{\mathrm{BH}^{-}} \sigma$ relation to the galaxies with $\sigma<100 \mathrm{~km} \mathrm{~s}^{-1}$ in Illustris ${ }^{14}$ and define overmassive and undermassive SMBHs similarly to what is done for massive galaxies. We find that the overmassiveness of SMBHs in low-mass galaxies correlates with the star formation history in ways similar to the massive galaxies in Illustris. As

\footnotetext{
${ }^{14}$ As discussed in Section 3, the slope of $M_{\mathrm{BH}^{-}} \sigma$ is shallower for low-mass galaxies in Martín-Navarro \& Mezcua (2018) but steeper in Illustris.
}

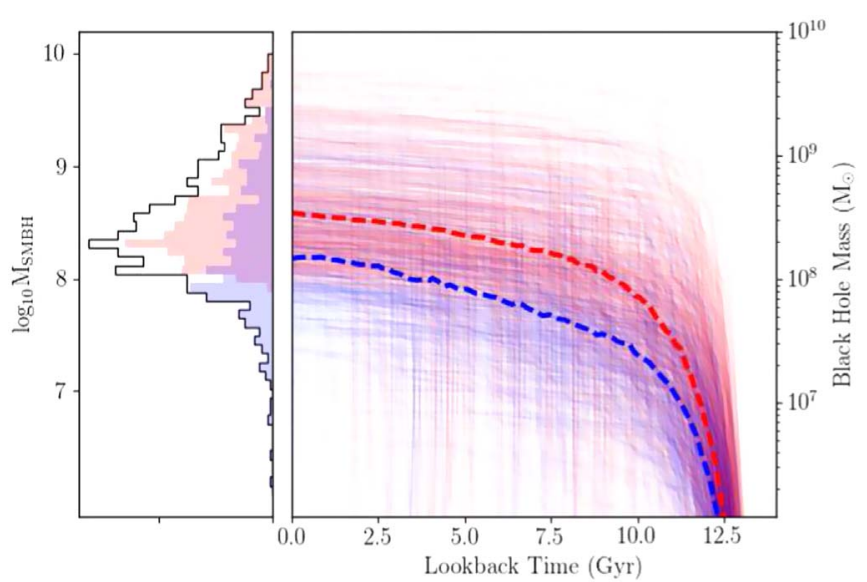

Figure 5. Main panel: the evolution of the $M_{\mathrm{BH}}$ (bottom) of galaxies with $\sigma>100 \mathrm{~km} \mathrm{~s}^{-1}$ in Illustris. Red shows the galaxies with overmassive SMBHs and blue shows galaxies with undermassive SMBHs. Faint lines show the trajectory of individual galaxies. Thick dashed lines connect the median values of the two populations at each lookback time. The dips (downward trend followed by a sudden increase) represent a misidentification of the main progenitor, which can occur during mergers (Rodriguez-Gomez et al. 2015). The small panel on the left shows the distribution of $M_{\mathrm{BH}}$ at $z=0$. Again, red shows the galaxies with overmassive SMBHs and blue shows galaxies with undermassive SMBHs. The black line shows the mass distribution of all the SMBHs in galaxies with $\sigma>100 \mathrm{~km} \mathrm{~s}^{-1}$ in Illustris. 

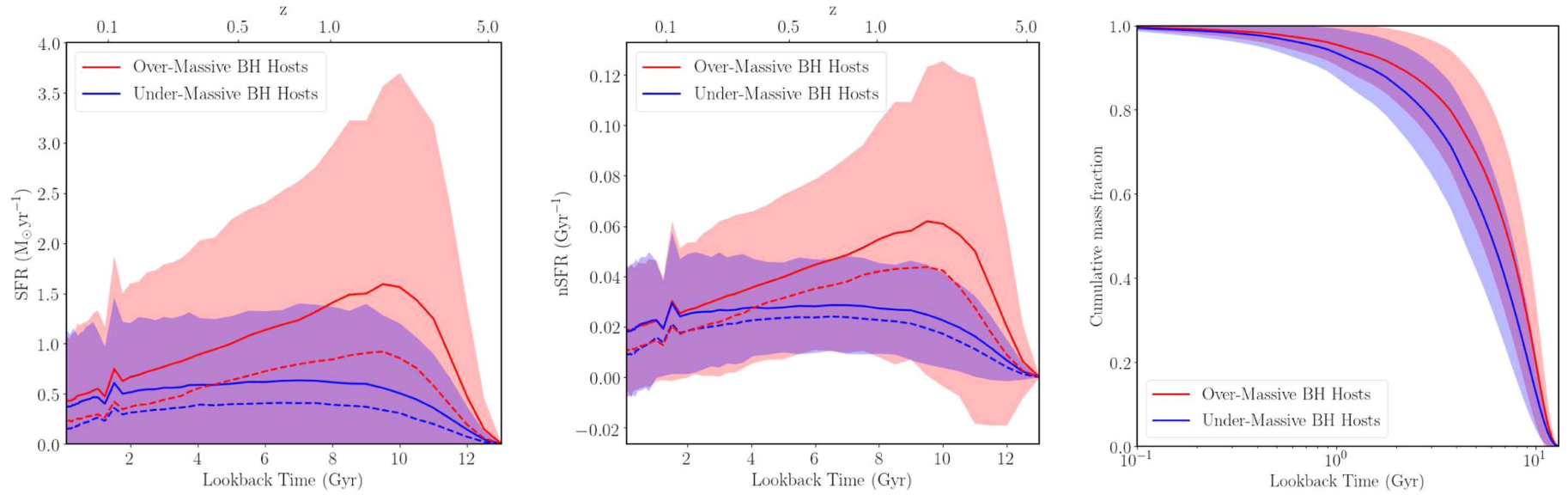

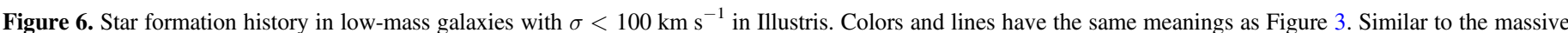

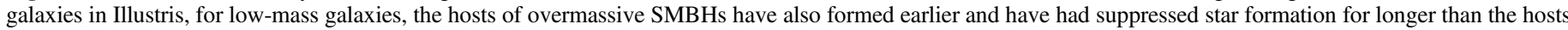
of undermassive SMBHs. This is different from Martín-Navarro \& Mezcua (2018), who find no such correlation for low-mass galaxies in the observed sample.

Figure 6 shows, the host galaxies of overmassive SMBHs have formed earlier, and their SFR is higher at higher redshift, but falls under the hosts of undermassive SMBHs. The nSFR of the two populations is comparable below $z \sim 2$. For low-mass galaxies, Martín-Navarro \& Mezcua (2018) use a fixed SDSS aperture, which is about $4.5 \mathrm{kpc}$. We have adopted the same aperture for our low-mass galaxies here, which is roughly their average $R_{e}$. Because simulated galaxies in Illustris tend to have larger sizes than the observed galaxies at given stellar mass (Bottrell et al. 2017), using an aperture with a fixed physical size may create a selection bias. We have experimented with $0.5 R_{e}$ and $2 R_{e}$, and found that the exact choice of aperture mainly affects the absolute level of SFR but does not change the basic trends.

Similar to the massive galaxies in Illustris, the vertical distance to the $M_{\mathrm{BH}^{-}}-\sigma$ relation for low-mass galaxies is also positively correlated with the Sérsic index. This can be seen from the right panels of Figure 4. However, the correlation between SMBH overmassiveness and baryon conversion efficiency for low-mass galaxies is different. Our statistical analysis suggests that they are positively correlated with each other, with $r=0.14$ and $\log _{10} p \sim-25$.

Simulated low-mass galaxies in Illustris have a stronger coupling between star formation history and black hole mass than what is reported in Martín-Navarro \& Mezcua (2018) for the observed low-mass galaxies. There are a few possible explanations for this disagreement. Perhaps AGN feedback in smaller galaxies should be modeled differently than in more massive systems. It is also possible that the stochasticity of $\mathrm{SMBH}$ feeding and feedback, which is poorly captured by the simulation, has a bigger impact in low-mass galaxies due to their shallower gravitational potential. The disagreement may also be related to uncertainties and biases in the observations. For the observed galaxies, the uncertainties are larger for the estimates of both $M_{\mathrm{BH}}$ and $\sigma$ at the low-mass end. The Martín-Navarro \& Mezcua (2018) sample is based on single-epoch black hole mass determination of active galaxies. It is also possible that there are unknown biases in the analysis of the star formation history for low-mass galaxies. In addition, the low-mass galaxies analyzed in the simulations are effectively selected based on their stellar masses at the low-mass end, while the selection bias in the observed sample is more complicated (Martín-Navarro \& Mezcua 2018; Shankar et al. 2019).
Recently, Dickey et al. (2019) conducted a spectroscopic study of a sample of isolated low-mass galaxies and concluded that AGN feedback is likely responsible for quenching these galaxies. Penny et al. (2018) and Manzano-King et al. (2019) find AGN-driven outflows from a sample of dwarf galaxies and evidence of suppressed star formation. These findings suggest that AGN feedback may still play a role in at least some lowmass galaxies and are consistent with what we find here based on the Illustris simulation. Note that in general, quenching in Illustris is found to be not efficient enough compared with the observations, whereas the level of star formation in TNG100 is in better agreement with the observations (Bluck et al. 2016; Nelson et al. 2018; Donnari et al. 2019). Our discussions here are mainly focused on the relative effect of feedback between different galaxies within the simulation, not the absolute level of quenching.

\subsection{SMBH Overmassiveness in TNG100}

We have performed the same analysis with the TNG100 data. The best linear fit $M_{\mathrm{BH}}-\sigma$ relation for massive galaxies in TNG100 is $\log _{10} M_{\mathrm{BH}}\left(M_{\odot}\right)=2.4 \log _{10} \sigma\left(\mathrm{km} \mathrm{s}^{-1}\right)+3.4$. As discussed in Section 4.1, in both observations and Illustris simulation, for galaxies with $\sigma>100 \mathrm{~km} \mathrm{~s}^{-1}$, the host galaxies of overmassive SMBHs have formed earlier and have been more quiescent at low redshift. This is not the case for TNG100.

Figure 7 shows that in TNG100, massive galaxies hosting overmassive SMBHs have always had a higher SFR than the hosts of undermassive black holes throughout their star formation history. They also have a slightly higher nSFR than the hosts of undermassive black holes at high redshift, but the nSFR of the two populations become similar at $z \lesssim 1$. The formation time of the hosts of overmassive SMBHs, measured by the cumulative mass fraction, is slightly earlier but the difference is barely noticeable. We have also compared the history of sSFR, and the hosts of undermassive SMBHs have higher sSFR than the hosts of overmassive SMBHs at low $z$, but only by a very small amount. At $z=0$, the median sSFR of the hosts of overmassive SMBHs is $\sim 3 \times 10^{-12} \mathrm{yr}^{-1}$ and for the hosts of undermassive SMBHs, it is $\sim 4 \times 10^{-12} \mathrm{yr}^{-1}$. For comparison, the median sSFRs of the hosts of over- and undermassive SMBHs in Illustris are $\sim 2 \times 10^{-12} \mathrm{yr}^{-1}$ and $\sim 4 \times 10^{-11} \mathrm{yr}^{-1}$, respectively. 

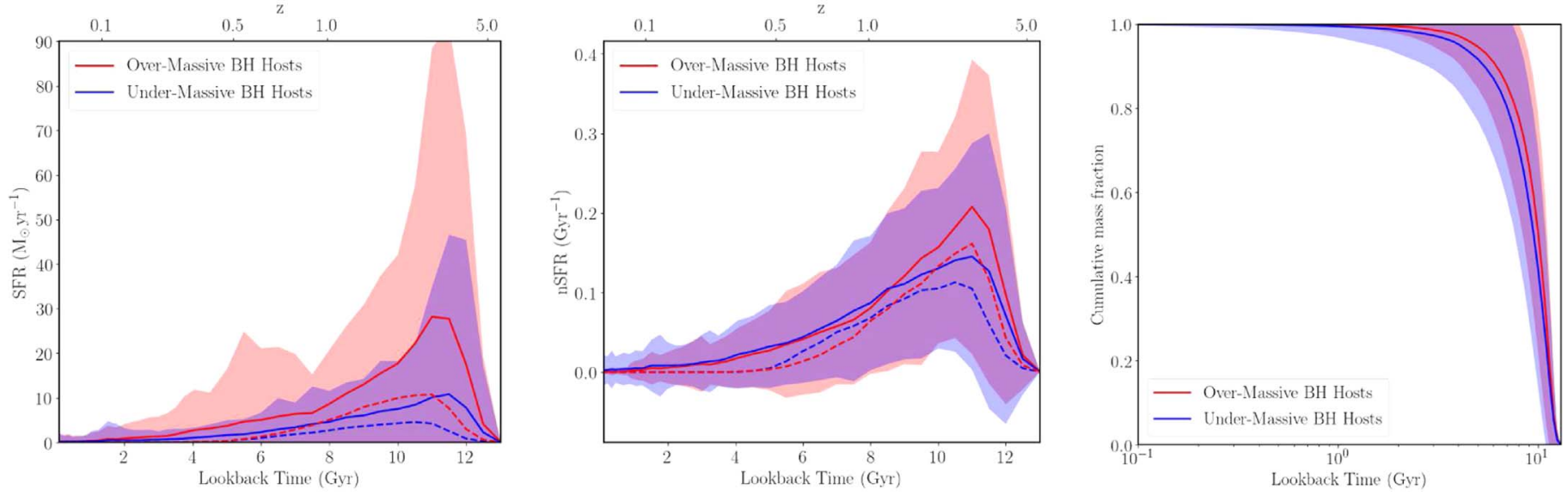

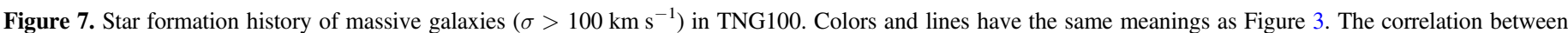

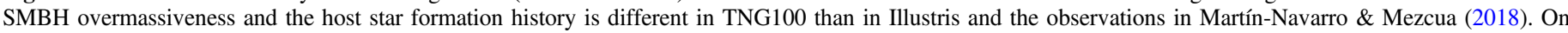

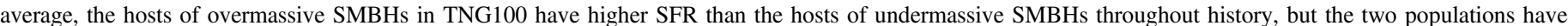
similar nSFR and cumulative star formation history.

To test the effect of selection bias against star-forming galaxies in Martín-Navarro et al. (2018), we have carried out the same experiment on TNG100 as we did for Illustris: we select TNG100 galaxies with sSFR $<10^{-11} \mathrm{yr}^{-1}$ and sSFR $<10^{-12} \mathrm{yr}^{-1}$, resulting in a subsample of 1780 and 1754 galaxies, respectively. Similar to the Illustris data, both TNG100 subsamples show the same trend as the full sample. Therefore, we use the full sample without an sSFR cut throughout the paper for TNG100 as well.

With a higher SFR throughout their history, the hosts of overmassive SMBHs should have a higher total stellar mass than the hosts of undermassive SMBHs. We have verified that the vertical distance to the average $M_{\mathrm{BH}}-\sigma$ relation is strongly correlated with $M_{*}$ in TNG100. This is consistent with our finding in Section 3 that in TNG100, $M_{\mathrm{BH}}$ and $M_{*}$ are very tightly correlated with each other. Thus, larger $M_{\mathrm{BH}}$ on the $M_{\mathrm{BH}}-\sigma$ relation translates to larger $M_{*}$.

Because a linear fit does not describe the $M_{\mathrm{BH}^{-}} \sigma$ relation in TNG100 very well (Figure 1), we have also tried to define the average $M_{\mathrm{BH}}-\sigma$ relation using higher-order polynomials. We have also tried to divide the data into bins based on $\sigma$ and use the median $M_{\mathrm{BH}}$ within $\sigma$ bins. All these experiments give the same results. We have also tried to choose a narrower range of $\sigma$. For example, we have selected only galaxies with $2.2<\log _{10} \sigma\left(\mathrm{km} \mathrm{s}^{-1}\right)<2.4$ (the very high-mass end avoiding the flattening part of the $M_{\mathrm{BH}}-\sigma$ relation), and the correlation remains the same. When we select galaxies with $1.9<\log _{10} \sigma\left(\mathrm{km} \mathrm{s}^{-1}\right)<2.1$ (the flattening part of $M_{\mathrm{BH}}-\sigma$ around the threshold black hole mass for the low-statemode feedback), we find that the hosts of overmassive SMBHs do have a slightly lower average SFR than the hosts of undermassive SMBHs at $z=0$. However, this suppression of star formation happened only $1 \mathrm{Gyr}$ ago, instead of several gigayears ago as is found in Illustris and the observed data.

Given the large uncertainties in recovering the star formation history of galaxies observationally, there is a possibility that the observed correlation in Martín-Navarro et al. (2018) is due to hidden biases. It is uncanny that Illustris would reproduce similar trends though. This is even more intriguing considering that Illustris intermediate- and high-mass galaxies have been demonstrated not to show the observed levels of "quiescence," and they exhibit overall smaller quenched or red fractions than the TNG populations (Vogelsberger et al. 2014a; Nelson et al. 2018;

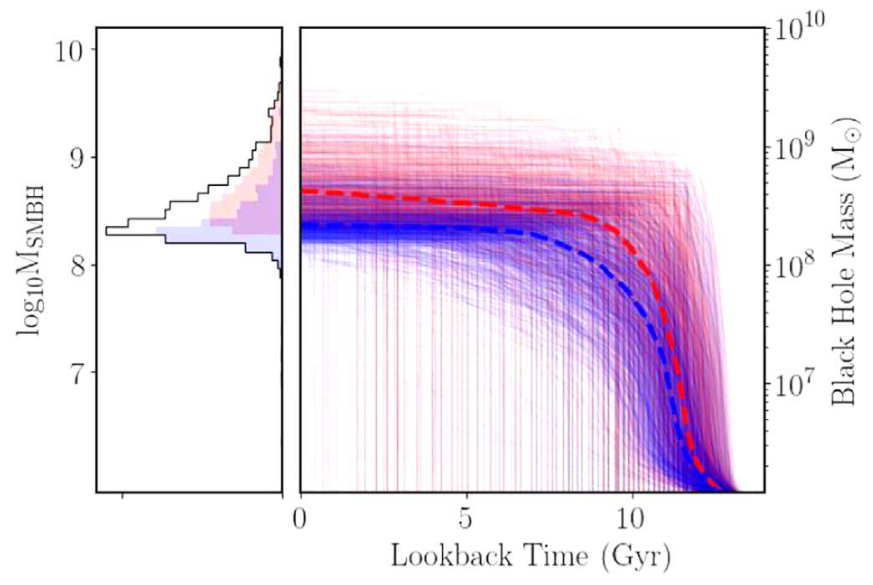

Figure 8. Main panel: the growth history of overmassive SMBHs (red) and undermassive SMBHs (blue) in TNG100. The left panel shows the distribution of $M_{\mathrm{BH}}$ at $z=0$. Thick dashed lines show the median values of the two populations. This is to be compared with Figure 5 for Illustris. Faint lines show the trajectory of individual galaxies and are slightly fainter than in Figure 5 for the clarity of the figure.

Donnari et al. 2019). More likely, TNG100 fails to recover the correlation between SMBH overmassiveness and quiescence.

One possible explanation for the discrepancy is the overly efficient feedback at $M_{\mathrm{BH}} \gtrsim 10^{8} M_{\odot}$ discussed previously. The massive galaxies with $\sigma>100 \mathrm{~km} \mathrm{~s}^{-1}$ have $M_{\mathrm{BH}}$ above this critical mass and are in the very effective pure kinetic feedback mode described in Section 2. The effect of overly efficient feedback at the threshold mass can also be seen in Figure 8, which shows the evolution of SMBHs in galaxies with $\sigma>100 \mathrm{~km} \mathrm{~s}^{-1}$ in TNG100. We see again the flattening of the history of individual galaxies at $M_{\mathrm{BH}} \gtrsim 10^{8} M_{\odot}$. Note that the actual transition black hole mass is slightly higher at higher redshift (between lookback time of 5 and $10 \mathrm{Gyr}$, the overdense "strip" turns upwards). This is simply because at higher redshift, black hole accretion rates tend to be higher, and the transition to low-state mode is harder (see Equation (1) and Weinberger et al. 2018).

The galaxies hosting overmassive SMBHs and the ones hosting undermassive SMBHs are less separated in TNG100 than in Illustris, and the distance between the median values is also smaller (Figure 5). The distribution of $M_{\mathrm{BH}}$ in TNG100 is 
narrower and more peaked near the critical mass than that in Illustris. All of these are consistent with the shallower slope of the $M_{\mathrm{BH}^{-}} \sigma$ relation in TNG100 discussed earlier, and the horizontal features on black hole scaling relations (Section 3).

Figure 8 also suggests that the overdensity around $M_{\mathrm{BH}} \gtrsim$ $10^{8} M_{\odot}$ already existed more than $8 \mathrm{Gyr}$ ago (corresponding to $z \sim 1$ ). In Illustris, this happens to be the time when hosts of undermassive SMBHs start to show higher average SFRs than the hosts of overmassive SMBHs. The very effective kinetic feedback in TNG100 around the critical black hole mass not only limits the growth of the SMBHs themselves, but also very effectively suppresses star formation of the host galaxies. It is likely that some galaxies with undermassive SMBHs (but still with $M_{\mathrm{BH}} \gtrsim 10^{8} M_{\odot}$ ) should have more late-time star formation, but end up producing too few young stars in TNG100. Another factor that contributes to the discrepancy between TNG100 and observations is that SMBHs also tend to be generally overly massive at $\log _{10} \sigma\left(\mathrm{km} \mathrm{s}^{-1}\right)<2.2$ (Section 3). Thus, we miss a population of galaxies with lower $M_{\mathrm{BH}}$ and low-level star formation (but not fully quenched) at low redshift. This is consistent with what we find in (Terrazas et al. 2020). We emphasize again that our discussions are focused on a specific aspect of the observations that compare the relative differences in the star formation history of galaxies related to their black hole masses. In general, TNG100 produces quenched galaxies in good agreement with the observations in terms of their absolute level of star formation (or suppression of star formation), while Illustris galaxies tend to have an SFR that is too high (Donnari et al. 2019).

Although the correlation between SMBH overmassiveness and quiescence in TNG100 is different from Illustris, for massive galaxies, SMBH overmassiveness correlates with baryon conversion efficiency and Sérsic indices in a similar way: galaxies with overmassive SMBHs tend to have a lower baryon conversion efficiency and a larger Sérsic index (Figure 9). However, this is not the case for low-mass galaxies in TNG100. As Figure 9 shows, for galaxies with $\sigma<100 \mathrm{~km} \mathrm{~s}^{-1}$, there is no obvious correlation between overmassiveness and Sérsic $n$ or baryon conversion efficiency. A Pearson's correlation analysis gives a coefficient of $r=-0.02$ and a $p$ value of 0.1 , suggesting no statistically significant correlations.

On the other hand, the star formation history of low-mass galaxies in TNG100 shows a very weak correlation with the overmassiveness of their SMBHs (Figure 10), very similar to TNG100 massive galaxies. In that regard, low-mass galaxies in TNG100 behave similarly to the observed low-mass galaxies in Martín-Navarro \& Mezcua (2018). However, we again note that the TNG100 $M_{\mathrm{BH}^{-}} \sigma$ relation differ from the observed relation in both its normalization and slope at the low-mass end. Low-mass galaxies in TNG100 also show a much tighter $M_{\mathrm{BH}}-M_{*}$ relation than the observed galaxies (Figure 1 and Section 3).

\subsection{Multidimensional SMBH Scaling Relations and the Effects of AGN Feedback}

Since the discovery of the $M_{\mathrm{BH}}-\sigma$ relation, much attention has been paid to its scatter (Ferrarese \& Merritt 2000; Gebhardt et al. 2000; Gültekin et al. 2009). It has been shown that the scatter in $M_{\mathrm{BH}^{-}} \sigma$ relation can be reduced by introducing a third parameter related to the property of the host galaxy (Marconi \& Hunt 2003; Barway \& Kembhavi 2007; Graham 2008). In other words, similar to elliptical galaxies, there are "fundamental planes" for supermassive black holes as well. Our study also suggests that
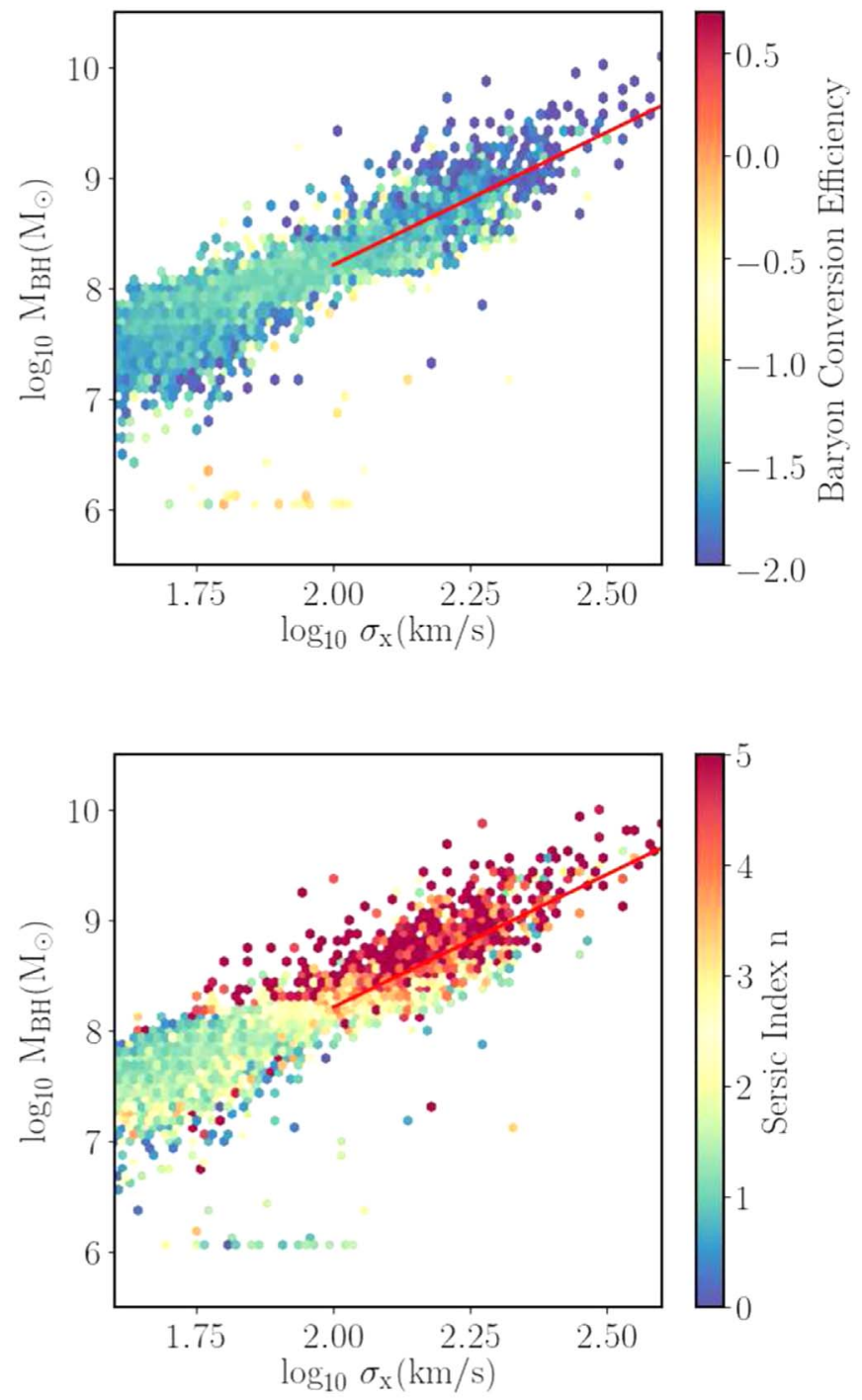

Figure 9. $M_{\mathrm{BH}}-\sigma$ relation weighted by baryon conversion efficiency (top) and Sérsic indices (bottom) in TNG100.

although the $M_{\mathrm{BH}}-\sigma$ relation is rather tight and is often considered "fundamental," it should not be viewed as a oneto-one correlation. Statistically, galaxies on the two sides of the mean relation have slightly different formation histories. In the Appendix, we show the $M_{\mathrm{BH}}-\sigma-n$ and $M_{\mathrm{BH}}-\sigma-$ eff (baryon conversion efficiency) planes for galaxies with $\sigma>100 \mathrm{~km} \mathrm{~s}^{-1}$ in both Illustris and TNG100. In all cases, adding a third parameter reduces the residuals of the fit. In reality, the evolution of SMBHs correlates with many properties of the host galaxies, and the most fundamental black hole correlation is likely not a linear correlation or a plane, but rather, a multidimensional manifold.

We also stress that the correlation between the overmassiveness of SMBHs and quiescence seen in Illustris and the observed data (Martín-Navarro et al. 2018) should not be simply attributed to "effective" AGN feedback (although it is certainly related). It is known that AGN feedback is not very effective in Illustris (in terms of shutting off late-time star formation) and is very effective for the massive galaxies in TNG100. Naively, one would expect to see a stronger correlation between the overmassiveness of SMBHs and quiescence in TNG100 than Illustris, especially given that the 

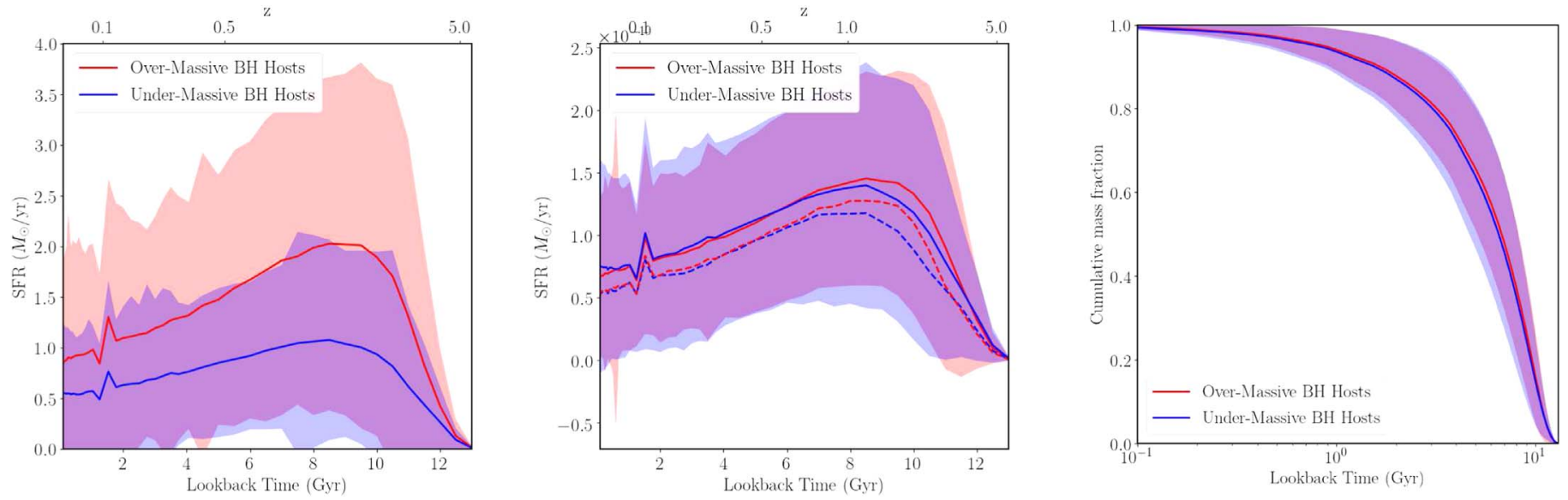

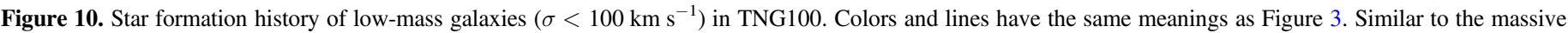

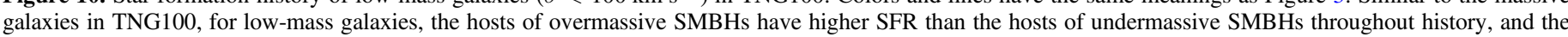
normalized star formation histories of the two populations are very similar.

effective kinetic feedback is linked to $M_{\mathrm{BH}}$ in TNG100 by design. Instead, the observed trend is seen more strongly in Illustris, which has less effective AGN feedback, but not TNG100 with more effective feedback. An important difference between AGN feedback in Illustris and TNG100 is that TNG100 feedback has more "features" (e.g., the switch between different feedback modes depends on $M_{\mathrm{SMBH}}$ ). Some $\mathrm{SMBH}$ properties or correlations are more sensitive to the sheer strength of AGN feedback, while some, such as the one we examined here, are more sensitive to how the implementation of AGN feedback is related to the properties of the host galaxies. Our analysis shows that the strength of feedback does not guarantee an imprint in $M_{\mathrm{BH}^{-}} \sigma$ versus star formation history. Black hole growth, star formation history, stellar mass, and $\sigma$ are related in complicated ways. As we have shown, the overmassiveness of SMBHs is related to many other properties of the host galaxies, including the Sérsic indices and the baryon conversion efficiency. The galaxies hosting overmassive SMBHs in Illustris not only formed their stars earlier, but have also formed the SMBHs earlier and have grown the dark matter halos earlier and bigger.

\section{Conclusions}

We have studied the correlations between SMBH and the properties of their host galaxies in the Illustris and TNG100 simulations, including the $M_{\mathrm{BH}}-\sigma$ relation, $M_{\mathrm{BH}}-M_{*}$ relation, and the relation between $M_{\mathrm{BH}}$ and the Sérsic index of the host galaxy. We have examined the correlation between SMBH overmassiveness (defined as the vertical distance to the average $M_{\mathrm{BH}}-\sigma$ relation) and the star formation history of the host galaxies in Illustris and TNG100. We have contrasted their outcomes with those determined by selected observational data sets, albeit taken at face value and without correcting for observational selection biases. The main findings are summarized as follows:

1. In both Illustris and TNG100, $M_{\mathrm{BH}}$ is correlated with $\sigma$, $M_{*}$, and the Sérsic index, similar to the observations. The $M_{\mathrm{BH}}-M_{*}$ relation is tighter in simulations than observations, especially in TNG100. In both Illustris and TNG100, the $M_{\mathrm{BH}^{-}}-\sigma$ relation and $M_{\mathrm{BH}^{-}}-n$ relation show an offset from the observed correlations. This is due to the fact that the simulated galaxies host somewhat overmassive black holes (by 0.2 dex for Illustris and 1.2 dex for TNG100 at $\log _{10} M_{*}\left(M_{\odot}\right)=10.2$ ), as well as may be too large (by up to $0.1 \mathrm{dex}$ at $M_{*}=10^{11} M_{\odot}$ ) and consequently have too small velocity dispersions (by up to $0.05 \mathrm{dex}$ at the same mass). We speculate that the offset at the low-mass end for TNG100 also has to do with the large SMBH seed mass combined with efficient growth (inefficient feedback) in the TNG100 quasar mode.

2. All the black hole scaling relations in TNG100 show horizontal features at $M_{\mathrm{BH}} \gtrsim 10^{8} M_{\odot}$. This is the critical mass that helps define the transition between quasarmode and low-state-mode feedback. If this transition has an $M_{\mathrm{BH}}$-dependent Eddington-ratio transition instead of a fixed fraction of the Eddington ratio, we may be able to find similar features in the observed data.

3. In Illustris, galaxies that host overmassive SMBHs (with $M_{\mathrm{BH}}$ above the mean $M_{\mathrm{BH}}-\sigma$ relation) on average have formed earlier (with higher star formation rate at high redshift) and have had lower star formation rates at low redshift compared with the hosts of undermassive SMBHs. This is in qualitative agreement with the findings in Martín-Navarro et al. (2018) for the observed massive galaxies with $\sigma>100 \mathrm{~km} \mathrm{~s}^{-1}$. For galaxies with $\sigma<100 \mathrm{~km} \mathrm{~s}^{-1}$, such a trend still exists in Illustris, but in the observations, star formation history does not show a clear correlation with the overmassiveness of SMBHs in low-mass galaxies (Martín-Navarro \& Mezcua 2018).

4. In TNG100, galaxies that host overmassive SMBHs on average also tend to have formed earlier, but the trend is very weak. Galaxies with overmassive SMBHs in TNG100 always have a higher average star formation rate than the hosts of undermassive SMBHs throughout their star formation history. SMBH overmassiveness strongly correlates with the $M_{*}$ of the host galaxies. The correlation between SMBH overmassiveness and the star formation history of the hosts is weaker in TNG100, possibly due to AGN feedback being too efficient at $M_{\mathrm{BH}} \gtrsim 10^{8} M_{\odot}$. This overly suppresses both the growth of the SMBHs themselves and star formation in these galaxies. This correlation reflects the interplay of black hole growth, star formation history, and galaxy structure 
in a complex way, even in models where AGN feedback is responsible for quiescence.

5. For all the galaxies we have studied with $\sigma>100 \mathrm{~km} \mathrm{~s}^{-1}$ in Illustris and TNG100, overmassiveness of SMBHs correlates positively with the Sérsic indices and negatively with the baryon conversion efficiency. In both Illustris and TNG100, Sérsic indices and baryon conversion efficiency can be used as a second parameter to reduce the amount of scatter in the $M_{\mathrm{BH}^{-}}-\sigma$ relation for massive galaxies. The correlations are different for galaxies with $\sigma<100 \mathrm{~km} \mathrm{~s}^{-1}$.

The physical properties of a galaxy are determined by its full formation history, which is extremely complicated. We should interpret correlations with caution as they do not necessarily suggest causality or at least not direct or simple causality. On the other hand, the complex interplay of physical processes in models with effective low-stat- mode AGN feedback can yield a diversity of observational signatures. These signatures are powerful and decisive probes of the implementation of diverse physical processes in hydro models. Therefore, observations of black hole-host galaxy relations are powerful discriminants between models, and with sufficient care can be used to guide refinement of these models.

We thank Dandan Xu for generously providing her catalogs. We thank Amy Reines and Alister Graham for sharing their observed data. We thank Eliot Quataert, Ena Choi, Kareem ElBadry, and Ignacio Martin-Navarro for helpful discussions. We acknowledge the technical support from the Scientific Computing Core of the Simons Foundation.

\section{Appendix}

As is shown in Section 4, in both Illustris and TNG100, the residual of the $M_{\mathrm{BH}}-\sigma$ relation correlates with the Sérsic index $n$ of the host galaxy, and the baryon conversion efficiency eff for galaxies with $\sigma>100 \mathrm{~km} \mathrm{~s}^{-1}$. It has been shown that the scatter in the observed $M_{\mathrm{BH}}-\sigma$ relation can be reduced by introducing a third parameter related to the property of the host galaxy (Marconi \& Hunt 2003; Barway \& Kembhavi 2007; Graham 2008). In Figure A1, we show the "fundamental planes" of $M_{\mathrm{BH}^{-}}-\sigma-n$ and $M_{\mathrm{BH}^{-}}-\sigma$-eff for galaxies with $\sigma>100 \mathrm{~km} \mathrm{~s}^{-1}$ in Illustris and TNG100.

The functional form we use for the least-squares fitting is a simple linear relation: $\log _{10} M_{\mathrm{BH}}\left(M_{\odot}\right)=C[0] \log _{10} \sigma\left(\mathrm{km} \mathrm{s}^{-1}\right)+$ $C[1] \log _{10} Y+C$ [2], where $Y$ is either $n$ or eff. There is no obvious reason to assume that these quantities should correlate with each other in this particular functional form, but for simplicity, we use a linear fit. In other words, we assume that these fundamental planes are flat. Table A1 summarizes the best-fitting parameters and the rms residuals, including those for the simple $M_{\mathrm{BH}^{-}}-\sigma$ relation without a third parameter. In all cases with a third parameter ( $n$ or eff), the $M_{\mathrm{BH}^{-}}-$relation is further tightened.
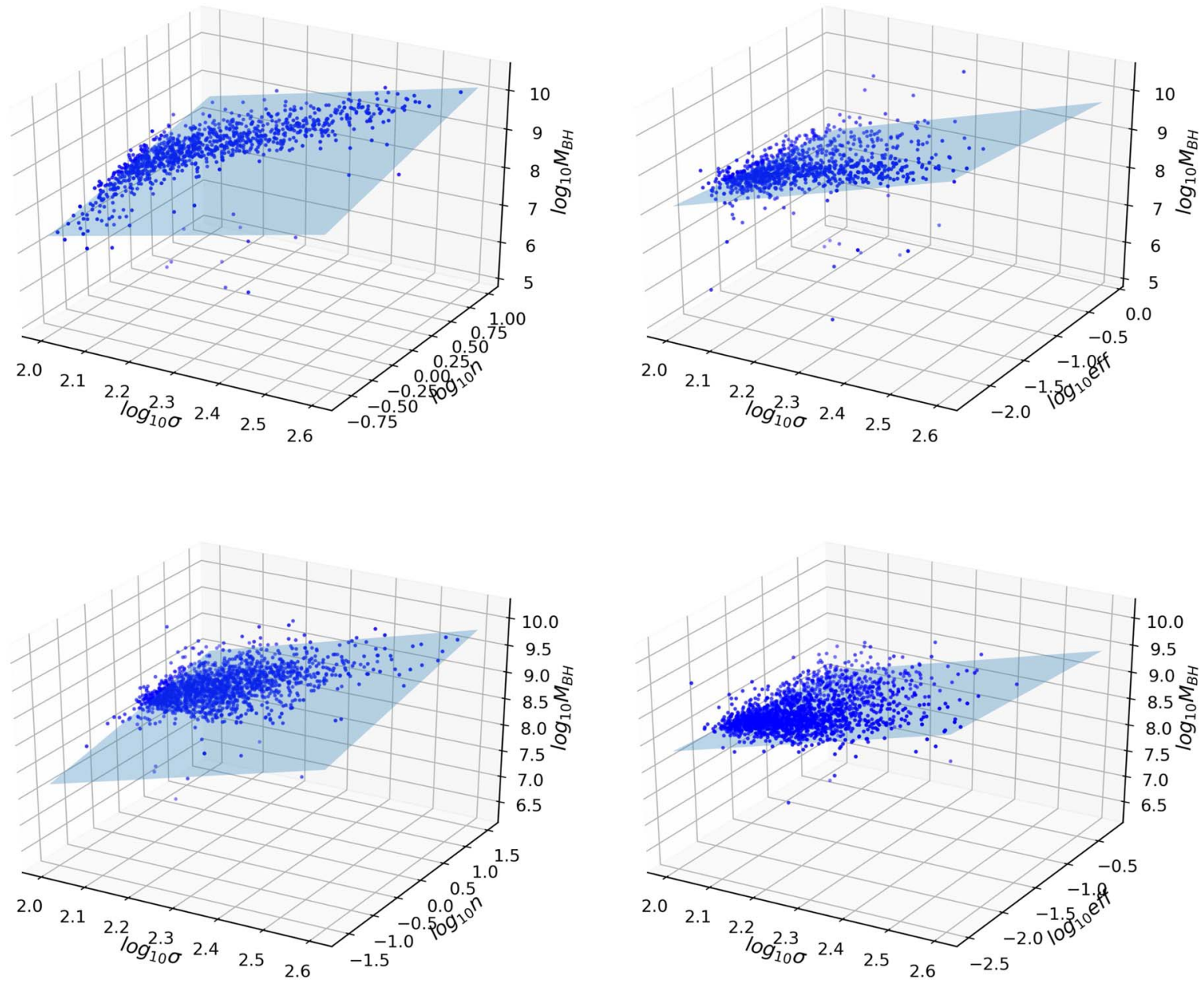

Figure A1. SMBH fundamental planes in Illustris (top) and TNG100 (bottom). Left panels show the $M_{\mathrm{BH}}-\sigma-n$ planes, where $n$ is the Sérsic index, and right panels show the $M_{\mathrm{BH}}-\sigma$-eff planes with eff being the baryon conversion efficiency. 
Table A1

Summary of Analysis Methods and Measurements of Galaxy Properties

\begin{tabular}{lllllc}
\hline \hline Relation & Simulation & $C[0]$ & $C[1]$ & $C[2]$ & rms Residual \\
\hline$M_{\mathrm{BH}^{-}-\sigma}$ & Illustris & 3.69 & $\cdots$ & 0.56 & 0.39 \\
\cline { 2 - 6 } & TNG100 & 2.40 & $\ldots$ & 3.41 & 0.22 \\
\hline$M_{\mathrm{BH}^{-}-\sigma-n}$ & Illustris & 2.62 & 0.65 & 2.68 & 0.36 \\
\cline { 2 - 6 } & TNG100 & 2.28 & 0.23 & 3.54 & 0.20 \\
\hline$M_{\mathrm{BH}}-\sigma-$ eff & Illustris & 3.51 & -0.21 & 0.63 & 0.38 \\
\cline { 2 - 6 } & TNG100 & 2.28 & -0.12 & 3.47 & 0.21 \\
\hline
\end{tabular}

Note. Parameters of $M_{\mathrm{BH}^{-}} \sigma$ relation and SMBH "fundamental planes" in Illustris and TNG100. In all cases, adding a third parameter ( $n$ or eff) reduces the rms residual of the $M_{\mathrm{BH}}-\sigma$ relation.

As Figure A1 shows, the $M_{\mathrm{BH}}-\sigma$ relation can be seen as a 2D projection of a more fundamental plane, but the two "fundamental planes" shown in Figure A1 are likely only projections of higher dimensional correlations. In fact, for both Illustris and TNG100, the smallest rms residuals are achieved when we include both Sérsic index $n$ and eff in the fitting. As is discussed in Section $4.2, M_{\mathrm{BH}}$ likely correlates with multiple parameters related to the host galaxy properties, and we have only explored a fraction of them in this work.

\section{ORCID iDs}

Yuan Li (ㄷ) https://orcid.org/0000-0001-5262-6150 Melanie Habouzit (10) https://orcid.org/0000-0003-4750-0187 Shy Genel $\odot$ https://orcid.org/0000-0002-3185-1540 Bryan A. Terrazas (i) https://orcid.org/0000-0001-5529-7305 Eric F. Bell (1) https://orcid.org/0000-0002-5564-9873 Annalisa Pillepich (1) https://orcid.org/0000-0003-1065-9274 Rainer Weinberger (1) https://orcid.org/0000-0001-6260-9709 Chung-Pei Ma (i) https://orcid.org/0000-0002-4430-102X Lars Hernquist (ㄴ) https://orcid.org/0000-0001-6950-1629

\section{References}

Abazajian, K., Adelman-McCarthy, J. K., Agüeros, M. A., et al. 2004, AJ, 128,502

Abazajian, K. N., Adelman-McCarthy, J. K., Agüeros, M. A., et al. 2009, ApJS, 182, 543

Anglés-Alcázar, D., Özel, F., \& Davé, R. 2013, ApJ, 770, 5

Arav, N., Borguet, B., Chamberlain, C., Edmonds, D., \& Danforth, C. 2013, MNRAS, 436, 3286

Baes, M., Verstappen, J., de Looze, I., et al. 2011, ApJS, 196, 22

Baldi, A., Forman, W., Jones, C., et al. 2009, ApJ, 707, 1034

Barway, S., \& Kembhavi, A. 2007, ApJL, 662, L67

Berrier, J. C., Davis, B. L., Kennefick, D., et al. 2013, ApJ, 769, 132

Blandford, R., Meier, D., \& Readhead, A. 2019, ARA\&A, 57, 467

Blanton, E. L., Randall, S. W., Clarke, T. E., et al. 2011, ApJ, 737, 99

Bluck, A. F. L., Mendel, J. T., Ellison, S. L., et al. 2016, MNRAS, 462, 2559

Booth, C. M., \& Schaye, J. 2009, MNRAS, 398, 53

Bottrell, C., Torrey, P., Simard, L., \& Ellison, S. L. 2017, MNRAS, 467, 2879

Bu, D.-F., Yuan, F., Gan, Z.-M., \& Yang, X.-H. 2016, ApJ, 818, 83

Bustamante, S., \& Springel, V. 2019, MNRAS, 490, 4133

Cano-Díaz, M., Maiolino, R., Marconi, A., et al. 2012, A\&A, 537, L8 Carniani, S., Marconi, A., Maiolino, R., et al. 2015, A\&A, 580, A102 Chartas, G., Brandt, W. N., \& Gallagher, S. C. 2003, ApJ, 595, 85 Cheung, E., Bundy, K., Cappellari, M., et al. 2016, Natur, 533, 504 Choi, E., Ostriker, J. P., Naab, T., \& Johansson, P. H. 2012, ApJ, 754, 125 Choi, E., Ostriker, J. P., Naab, T., Oser, L., \& Moster, B. P. 2015, MNRAS, 449, 4105

Churazov, E., Sazonov, S., Sunyaev, R., et al. 2005, MNRAS, 363, L91
Costa, T., Rosdahl, J., Sijacki, D., \& Haehnelt, M. G. 2018, MNRAS, 479, 2079

Davé, R., Anglés-Alcázar, D., Narayanan, D., et al. 2019, MNRAS, 486, 2827

Davis, B. L., Graham, A. W., \& Cameron, E. 2019, ApJ, 873, 85

Dickey, C., Geha, M., Wetzel, A., \& El-Badry, K. 2019, ApJ, 884, 180

Donnari, M., Pillepich, A., Nelson, D., et al. 2019, MNRAS, 485, 4817

Dubois, Y., Devriendt, J., Slyz, A., \& Teyssier, R. 2010, MNRAS, 409, 985

Dunn, J. P., Bautista, M., Arav, N., et al. 2010, ApJ, 709, 611

Dunn, R. J. H., \& Fabian, A. C. 2006, MNRAS, 373, 959

Dutton, A. A., Conroy, C., van den Bosch, F. C., et al. 2011, MNRAS, 416, 322

Fabian, A. C. 1994, ARA\&A, 32, 277

Fabian, A. C. 2012, ARA\&A, 50, 455

Fabian, A. C., Sanders, J. S., Taylor, G. B., et al. 2006, MNRAS, 366, 417

Ferrarese, L., \& Merritt, D. 2000, ApJL, 539, L9

Feruglio, C., Fiore, F., Carniani, S., et al. 2015, A\&A, 583, A99

Fragile, P. C., Blaes, O. M., Anninos, P., \& Salmonson, J. D. 2007, ApJ, 668, 417

Gallazzi, A., Charlot, S., Brinchmann, J., \& White, S. D. M. 2006, MNRAS, 370, 1106

Gebhardt, K., Bender, R., Bower, G., et al. 2000, ApJL, 539, L13

Genel, S., Nelson, D., Pillepich, A., et al. 2018, MNRAS, 474, 3976

Genel, S., Vogelsberger, M., Springel, V., et al. 2014, MNRAS, 445, 175

Graham, A. W. 2008, ApJ, 680, 143

Graham, A. W., \& Driver, S. P. 2007, ApJ, 655, 77

Graham, A. W., Erwin, P., Caon, N., \& Trujillo, I. 2001, ApJL, 563, L11

Graham, A. W., \& Scott, N. 2013, ApJ, 764, 151

Greene, J. E., Peng, C. Y., Kim, M., et al. 2010, ApJ, 721, 26

Gültekin, K., Richstone, D. O., Gebhardt, K., et al. 2009, ApJ, 698, 198

Guo, F., \& Mathews, W. G. 2011, ApJ, 728, 121

Habouzit, M., Genel, S., Somerville, R. S., et al. 2019, MNRAS, 484, 4413

Hirschmann, M., Khochfar, S., Burkert, A., et al. 2010, MNRAS, 407, 1016

Hopkins, P. F., \& Elvis, M. 2010, MNRAS, 401, 7

Hopkins, P. F., Wetzel, A., Kereš, D., et al. 2018, MNRAS, 480, 800

Jahnke, K., \& Macciò, A. V. 2011, ApJ, 734, 92

Jiang, J., Walton, D. J., Parker, M. L., \& Fabian, A. C. 2018, MNRAS, 481, 639

Jiang, Y.-F., Stone, J., \& Davis, S. W. 2019, ApJ, 885, 144

Kellermann, K. I., Condon, J. J., Kimball, A. E., Perley, R. A., \& Ivezić, Ž. 2016, ApJ, 831, 168

Khalatyan, A., Cattaneo, A., Schramm, M., et al. 2008, MNRAS, 387, 13

Kormendy, J. 1993, in The Nearest Active Galaxies, ed. J. Beckman, L. Colina, \& H. Netzer (Madrid: Consejo Superior de Investigaciones Cientificas), 197

Kormendy, J., \& Gebhardt, K. 2001, in AIP Conf. Ser. 586, 20th Texas Symp. on Rrelativistic Astrophysics, ed. J. C. Wheeler \& H. Martel (Melville, NY: AIP), 363

Kormendy, J., \& Ho, L. C. 2013, ARA\&A, 51, 511

Li, Y., \& Bryan, G. L. 2014, ApJ, 789, 153

Li, Y., Bryan, G. L., Ruszkowski, M., et al. 2015, ApJ, 811, 73

Liu, C., Yuan, F., Ostriker, J. P., Gan, Z., \& Yang, X. 2013, MNRAS, 434, 1721

Magorrian, J., Tremaine, S., Richstone, D., et al. 1998, AJ, 115, 2285

Manzano-King, C., Canalizo, G., \& Sales, L. 2019, ApJ, 884, 54

Marconi, A., \& Hunt, L. K. 2003, ApJL, 589, L21

Marinacci, F., Vogelsberger, M., Pakmor, R., et al. 2018, MNRAS, 480, 5113

Martín-Navarro, I., Brodie, J. P., Romanowsky, A. J., Ruiz-Lara, T., \& van de Ven, G. 2018, Natur, 553, 307

Martín-Navarro, I., Brodie, J. P., van den Bosch, R. C. E., Romanowsky, A. J., \& Forbes, D. A. 2016, ApJL, 832, L11

Martín-Navarro, I., \& Mezcua, M. 2018, ApJL, 855, L20

Martizzi, D., Quataert, E., Faucher-Giguère, C.-A., \& Fielding, D. 2019, MNRAS, 483, 2465

McAlpine, S., Bower, R. G., Harrison, C. M., et al. 2017, MNRAS, 468, 3395

McConnell, N. J., \& Ma, C.-P. 2013, ApJ, 764, 184

McNamara, B. R., \& Nulsen, P. E. J. 2007, ARA\&A, 45, 117

Merritt, D., \& Ferrarese, L. 2001, MNRAS, 320, L30

Moe, M., Arav, N., Bautista, M. A., \& Korista, K. T. 2009, ApJ, 706, 525

Mutlu-Pakdil, B., Seigar, M. S., Hewitt, I. B., et al. 2018, MNRAS, 474, 2594

Naiman, J. P., Pillepich, A., Springel, V., et al. 2018, MNRAS, 477, 1206

Nelson, D., Pillepich, A., Springel, V., et al. 2018, MNRAS, 475, 624

Ocvirk, P., Pichon, C., Lançon, A., \& Thiébaut, E. 2006, MNRAS, 365, 74

Omma, H., Binney, J., Bryan, G., \& Slyz, A. 2004, MNRAS, 348, 1105

Pakmor, R., Springel, V., Bauer, A., et al. 2016, MNRAS, 455, 1134

Park, K., \& Ricotti, M. 2012, ApJ, 747, 9

Penny, S. J., Masters, K. L., Smethurst, R., et al. 2018, MNRAS, 476, 979 
Pillepich, A., Nelson, D., Hernquist, L., et al. 2018a, MNRAS, 475, 648 Pillepich, A., Springel, V., Nelson, D., et al. 2018b, MNRAS, 473, 4077

Proga, D. 2007, in Astronomical Society of the Pacific Conference Series, Vol. 373, The Central Engine of Active Galactic Nuclei, ed. L. C. Ho \& J.-W. Wang, 267

Qiu, Y., Bogdanovic, T., Li, Y., Park, K., \& Wise, J. H. 2019, ApJ, 877, 47

Rafferty, D. A., McNamara, B. R., Nulsen, P. E. J., \& Wise, M. W. 2006, ApJ, 652,216

Reines, A. E., \& Volonteri, M. 2015, ApJ, 813, 82

Rodriguez-Gomez, V., Genel, S., Vogelsberger, M., et al. 2015, MNRAS, 449, 49

Rodriguez-Gomez, V., Snyder, G. F., Lotz, J. M., et al. 2019, MNRAS, 483, 4140

Savorgnan, G. A. D. 2016, ApJ, 821, 88

Schawinski, K., Thomas, D., Sarzi, M., et al. 2007, MNRAS, 382, 1415

Schaye, J., Crain, R. A., Bower, R. G., et al. 2015, MNRAS, 446, 521

Seigar, M. S., Kennefick, D., Kennefick, J., \& Lacy, C. H. S. 2008, ApJL, 678, L93

Shankar, F., Bernardi, M., Richardson, K., et al. 2019, MNRAS, 485, 1278

Sijacki, D., Vogelsberger, M., Genel, S., et al. 2015, MNRAS, 452, 575

Snyder, G. F., Torrey, P., Lotz, J. M., et al. 2015, MNRAS, 454, 1886

Springel, V. 2010, MNRAS, 401, 791

Springel, V., di Matteo, T., \& Hernquist, L. 2005, MNRAS, 361, 776

Springel, V., Pakmor, R., Pillepich, A., et al. 2018, MNRAS, 475, 676
Tchekhovskoy, A., Narayan, R., \& McKinney, J. C. 2011, MNRAS, 418, L79 Terrazas, B. A., Bell, E. F., Henriques, B. M. B., et al. 2016, ApJL, 830, L12 Terrazas, B. A., Bell, E. F., Pillepich, A., et al. 2020, MNRAS, 493, 1888

Tombesi, F., Meléndez, M., Veilleux, S., et al. 2015, Natur, 519, 436

Tremaine, S., Gebhardt, K., Bender, R., et al. 2002, ApJ, 574, 740

van den Bosch, R. C. E. 2016, ApJ, 831, 134

Vazdekis, A., Sánchez-Blázquez, P., Falcón-Barroso, J., et al. 2010, MNRAS, 404, 1639

Vogelsberger, M., Genel, S., Sijacki, D., et al. 2013, MNRAS, 436, 3031

Vogelsberger, M., Genel, S., Springel, V., et al. 2014a, MNRAS, 444, 1518

Vogelsberger, M., Genel, S., Springel, V., et al. 2014b, Natur, 509, 177

Wang, Y., Vogelsberger, M., Xu, D., et al. 2020, MNRAS, 491, 5188

Weinberger, R., Springel, V., Hernquist, L., et al. 2017, MNRAS, 465, 3291

Weinberger, R., Springel, V., Pakmor, R., et al. 2018, MNRAS, 479, 4056

Wilson, A. S., \& Colbert, E. J. M. 1995, ApJ, 438, 62

Wise, M. W., McNamara, B. R., Nulsen, P. E. J., Houck, J. C., \& David, L. P. 2007, ApJ, 659, 1153

Woo, J.-H., Schulze, A., Park, D., et al. 2013, ApJ, 772, 49

Xie, F.-G., Yuan, F., \& Ho, L. C. 2017, ApJ, 844, 42

Xu, D., Springel, V., Sluse, D., et al. 2017, MNRAS, 469, 1824

Xu, D., Zhu, L., Grand, R., et al. 2019, MNRAS, 489, 842

Yang, H.-Y. K., \& Reynolds, C. S. 2016, ApJ, 829, 90

Yuan, F., \& Narayan, R. 2014, ARA\&A, 52, 529

Yuan, F., Yoon, D., Li, Y.-P., et al. 2018, ApJ, 857, 121 